\title{
Capacitance, induced charges, and bound states of biased carbon nanotube systems
}

\author{
Pawel Pomorski, ${ }^{1}$ Lars Pastewka, ${ }^{1}$ Christopher Roland, ${ }^{1}$ Hong Guo,${ }^{2}$ and Jian Wang ${ }^{3}$ \\ ${ }^{1}$ Department of Physics, The North Carolina State University, Raleigh, North Carolina 27695-8202, USA \\ ${ }^{2}$ Center for the Physics of Materials and Department of Physics, McGill University, Montreal, PQ, Canada H3A 2T8 \\ ${ }^{3}$ Department of Physics, The University of Hong Kong, Pokfulam Road, Hong Kong, China
}

(Received 7 October 2003; published 22 March 2004)

\begin{abstract}
Although it has long been known that the classical notions of capacitance need modification at the nanoscale, in order to account for important quantum effects, very few first-principles investigations of these properties exist for any real material systems. Here we present the results of a large-scale ab initio investigation of the capacitance properties of carbon nanotube systems. The simulations are based on a recently developed real-space nonequilibrium Green's-function approach, with special attention being paid to the treatment of the bound states present in the system. In addition, use has been made of a symmetry decomposition scheme for the charge density. This is needed both to speed up the calculations and in order to study the origins of the induced charges. Specific systems investigated include two and three nested nanotube shells, the insertion of a capped nanotube into another, a connected $(12,0) /(6,6)$ nanotube junction, and the properties of a nanotube acting as a probe over a flat aluminum surface. First-principles estimates of the capacitance matrix coefficients for all these systems are provided, along with a discussion of the quantum corrections. For the case of the nanotube junction, the numerical value of the capacitance is sufficiently high, as to be useful for future device applications.
\end{abstract}

DOI: 10.1103/PhysRevB.69.115418

PACS number(s): 73.61.Wp, 72.80.Rj

\section{INTRODUCTION}

Within classical electrostatics, the capacitance is a measure of a conductor's ability to store charge. ${ }^{1}$ The classical capacitance coefficients $C_{\alpha \beta}$ of a set of conductors are defined by $Q_{\alpha}=\Sigma_{\beta} C_{\alpha \beta} V_{\beta}$, which gives the accumulation of charge $Q_{\alpha}$ on conductor $\alpha$ in response to a change in the electrostatic potential $V_{\beta}$ on conductor $\beta$. The classical capacitance is purely a geometric quantity, and depends only on the shape and the spatial arrangements of the conductors and on the presence of any dielectric medium. A typical calculation of the conductance coefficients entails the solving of Poisson's equation for a series of conductors subject to the appropriate boundary conditions.

Central to the classical notion of capacitance is that of a set of well-defined conductors, with zero electric field in their bulk. These assumptions, however, typically break down at the nanometer length scale, when the screening length of the material becomes comparable to the dimensions of the system. In this case, conductors may no longer be equipotential surfaces, and the classical notion of capacitance has to be generalized to that of the electrochemical capacitance, where each conductor is connected to an electron reservoir with an electrochemical potential $\mu .^{2-6}$ Then, the selfconsistent charge variation $d Q_{\alpha}$ on conductor $\alpha$, when the electrochemical potential of the reservoir connected to conductor $\beta$ is changed by a small amount $d \mu_{\beta}$ with respect to some reference potential, is given by ${ }^{3}$

$d Q_{\alpha}=\sum_{\beta} C_{\alpha \beta}\left(d \mu_{\beta} / e\right)+\sum_{\beta \gamma} C_{\alpha \beta \gamma}\left(d \mu_{\beta} / e\right)\left(d \mu_{\gamma} / e\right)+\cdots$

In contrast to the classical case, there is no reason for the charge accumulation to be linear at a given finite-bias volt- age. Hence, $C_{\alpha \beta}$ and $C_{\alpha \beta \gamma}$ represent the first linear and nonlinear capacitance matrix coefficients, respectively. These coefficients take quantum effects into account, and may differ considerably from their classical counterparts. ${ }^{5,6}$ Note also that because conductors are no longer equipotential surfaces, the capacitance coefficients may no longer be calculated with geometrical techniques only. Instead, one has to investigate the electron dynamics and the induced rearrangement of charge in response to a change in the electrochemical potential, so that the concept of the electrochemical capacitance is intimately related to that of quantum transport. ${ }^{3,4,7,8}$

Although the theory of the quantum capacitance is almost a decade old, ${ }^{3}$ there have been few first-principles calculations of these properties for any real material systems. However, the recent advent of molecular electronic systems has given new urgency towards understanding fundamental problems of this type. In this paper, we present such an analysis for prototypical carbon nanotube systems, using a recently developed real-space nonequilibrium Green's-function formalism. ${ }^{9}{ }^{10}$ Specifically, the capacitance behavior of nanotube shells, the insertion of one nanotube into another, a metal-metal nanotube junction, and a nanotube acting as a probe over a flat aluminum surface will all be discussed.

We have focused on carbon nanotubes because of the very important role that this material system plays in the emerging field of nanotechnology. ${ }^{11}$ Depending on their helicity, carbon nanotubes are either metals or semiconductors, whichalong with their unique mechanical properties-makes them an ideal system for exploring quantum transport at the nanometer length scale. Indeed, a number of prototypical carbon nanotube-based devices with outstanding characteristics have already been produced, and their properties explored both experimentally ${ }^{12}$ and theoretically. ${ }^{13}$ The majority of 
these theoretical investigations have focused on the conductance and the current-voltage $(I-V)$ characteristics of nanotube devices. To date, there have been relatively few studies of the other transport properties such as the capacitance and self-inductance, ${ }^{14-17}$ which are all properties that depend on the induced rearrangement of charge, rather than on the direct flow of current. A good understanding of these properties is of course important both from a fundamental and a technological viewpoint. Capacitance properties are central to the workings of nanotubes as scanning probes, ${ }^{15}$ memory devices, their ability to store charge, and in understanding the dynamic response of nanotubes to externally applied alternating current (ac) fields. ${ }^{14,17}$

A short outline of this paper is as follows. In the following section, we briefly review the methodology which is based on $a b$ initio simulations using a real-space nonequilibrium Green's-function formalism. Section III presents a symmetry analysis of carbon nanotube systems, which is important both for speeding up the numerical aspects of the problem and for an analysis of the induced charge. This aspect is also important for technical reasons in order to properly deal with the bound states present in the system. Capacitance results for the different carbon nanotube systems are given in Sec. IV, while Sec. V is reserved for the final discussion and conclusions. Finally, we have relegated to Appendix A an outline of the calculations of the quantum corrections within the linear response regime.

\section{METHODOLOGY}

Our numerical investigations are based on a recently developed $a b$ initio formalism, ${ }^{9,10}$ which combines the Keldysh nonequilibrium Green's-function theory ${ }^{8,18,19}$ (NEGF) with real-space density-functional theory (DFT) simulations. As the details of this technique are somewhat technical and have been given elsewhere, we restrict ourselves here to a brief summary, and otherwise refer the interested reader to Refs. 9 and 10 for more details. To date, this method has been applied to an ever growing number of physical systems, including fullerenes, ${ }^{20,21}$ metallic nanowires, ${ }^{22,23}$ nanotube systems, ${ }^{17,24}$ clusters, ${ }^{25}$ and select organic compounds ${ }^{26,27}$ in a two-probe geometry. Roughly speaking, the main advantages of the NEGF-DFT approach are (i) it enables a proper treatment of the open-boundary conditions for a quantum system under a bias voltage; (ii) a fully atomistic treatment of the electrodes; and (iii) a self-consistent calculation of the charge density via NEGF, thereby incorporating the effect of both the scattering and the bound states in the system. Moreover, because of the extensive use of real-space grids, the entire procedure may be parallelized enabling the treatment of large systems.

Although the NEGF-DFT code has generally been used to calculate the $I-V$ characteristics of two-probe devices, ${ }^{9,10,21-27}$ it is both the electrostatic potential and the self-consistent charge density that are the two most important quantities for the calculation of the quantum capacitance. Calculation of the electrostatic potential is carried out by standard multigrid techniques, ${ }^{10,28}$ while the calculation of the charge density is based on the following consid- erations. The electronic states of the atoms are modeled using a linear combination of atomic orbitals ${ }^{29}$ with $\left\{\phi_{\nu}\right\}=\left\{\phi_{s}, \phi_{p_{x}}, \phi_{p_{y}}, \phi_{p_{z}}\right\}$ (Ref. 30) and standard pseudopotentials. ${ }^{31}$ The Kohn-Sham eigenstates of the system, with a shifted Hartree potential at the boundary between the leads and the central scattering region, ${ }^{9,10}$ are then expanded in terms of this basis:

$$
\Psi^{i}=\sum_{\nu} c_{\nu}^{i} \phi_{\nu}\left(\mathbf{r}-\mathbf{R}_{I}\right) \quad\left(\nu=s, p_{x}, p_{y}, p_{z}\right),
$$

and $I$ is an index for the atom number. The Kohn-Sham equation may then be transformed into a standard matrix problem: ${ }^{7}$

$$
H_{\mu \nu} c_{\nu}^{i}=E^{i} S_{\mu \nu} c_{\nu}^{i},
$$

with $H_{\mu \nu}$ and $S_{\mu \nu}$ representing the Hamiltonian and overlap matrix elements between the orbitals located at different sites, respectively. The information about the electron occupation and the charge density is contained in the density matrix $\hat{\rho}$, which is used to calculate the real-space density $\rho(\mathbf{r})$ :

$$
\rho(\mathbf{r})=\sum_{\mu \nu} \phi_{\mu}^{*}\left(\mathbf{r}-\mathbf{R}_{I}\right) \hat{\rho}_{\mu \nu} \phi_{\nu}\left(\mathbf{r}-\mathbf{R}_{J}\right),
$$

and the number of electrons within a given region,

$$
N_{\text {region }}=\operatorname{Tr}[\hat{\rho} S]_{\text {region }} .
$$

The NEGF-DFT formalism calculates both $H(\hat{\rho})$ and $\hat{\rho}$ selfconsistently to at least $10^{-3} \mathrm{eV}$ via standard iteration procedures. For the two-probe geometry, the NEGF theory gives the density matrix as

$$
\hat{\rho}=\frac{-i}{2 \pi} \int d E G^{<}(E)=\frac{-i}{2 \pi} \int d E G^{R} \Sigma^{<} G^{A},
$$

with $G^{R, A}$ denoting the retarded/advanced Green's function of the device as a function of energy $E$ and $\Sigma^{<}=$ $-2 i \operatorname{Im}\left(f_{l} \Sigma^{l}+f_{r} \Sigma^{r}\right)$ the lesser self-energy of the system. Here $\Sigma^{l, r}$ represent the self-energy due to the coupling to the left and right electrodes, respectively, and $f_{l, r}(E)$ the corresponding electron distribution function giving the occupation of each of the eigenstates of the electrodes. These quantities are all evaluated by standard means. ${ }^{9,10,32}$ For discussion purposes, let us view a device to be a very large (due to leads) but finite system, then $G^{R}$ may be expressed in terms of the eigenstates of the Hamiltonian $\left\{\Psi^{n}\right\}$ and their energies $\left\{E_{n}\right\}$ :

$$
G^{R}(E)=\left[H-E S-\Sigma^{l}-\Sigma^{r}\right]^{-1} \sim \lim _{\eta \rightarrow 0} \sum_{n} \frac{\left|\Psi^{n}\right\rangle\left\langle\Psi^{n}\right|}{E-E_{n}+i \eta} .
$$

For a system at equilibrium, $\mu_{l}=\mu_{r}=\mu$, all the states below this potential will be filled so that $f_{l}(E)=f_{r}(E)=1$. For this case,

$$
\operatorname{Re}\left[G^{<}(E)\right]=2 \operatorname{Im}\left[G^{R}(E)\right] .
$$




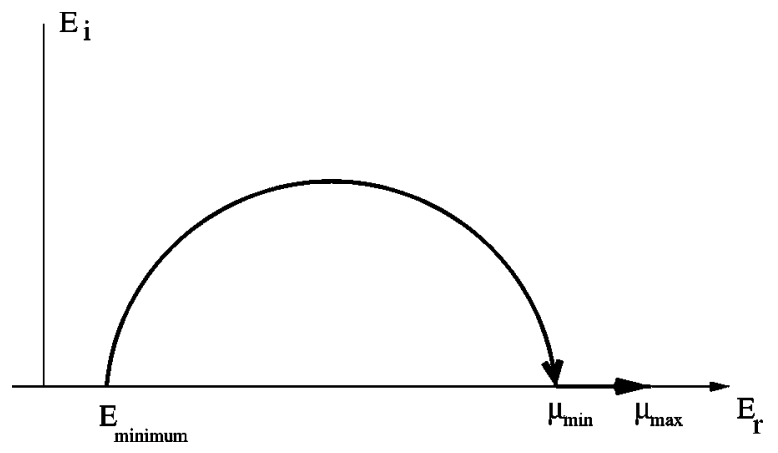

FIG. 1. Integration pathway in the complex plane used to evaluate $\hat{\rho}$, i.e., Eq. (9).

The nonequilibrium density matrix $\hat{\rho}$ may then be rewritten as

$$
\hat{\rho}=\frac{2}{\pi} \operatorname{Im}\left[\int_{-\infty}^{\mu_{\min }} d E G^{R}(E)\right]+\frac{1}{\pi} \operatorname{Re}\left[\int_{\mu_{\min }}^{\mu_{\max }} d E G^{<}(E)\right],
$$

where $\mu_{\text {min }}=\min \left(\mu_{l}+V_{l}, \mu_{r}+V_{r}\right)$ and $\mu_{\max }=\max \left(\mu_{l}+V_{l}, \mu_{r}\right.$ $\left.+V_{r}\right) .{ }^{10}$ Generally speaking, the direct evaluation of this integral contains contributions from both the scattering states-i.e., eigenstates with a continuous spectrum which correspond to electrons with wave functions extending infinitely into the leads - and bound states, which are states of discrete energy with the wave function localized in the central scattering region and decaying into the leads. Bound states can arise in a number of ways. For instance, they may arise when the molecules in the central scattering region have molecular states with energies below the propagating threshold of the leads, ${ }^{9}$ or when there are band gaps present in both of the lead electrodes. Bound states may also appear because of mismatches in the symmetries of the wave functions, as will be further discussed in Sec. IV. From Eq. (7), one can infer that $G^{R}$ has poles near $E=E_{m}-i \eta$, where $\left\{E_{m}\right\}$ are the discrete energies of the bound states. Since these poles lie below the real energy axis in the complex plane, $G^{R}$ is analytic above the real axis. Thus, a convenient way of dealing with Eq. (9) is to integrate the first term along a semicircle in the upper half of the complex plane starting from some minimum energy that lies below all the states, and ending on the real axis at $\mu_{\text {min }}$, as shown in Fig. 1. Numerically very accurate integration is achieved by means of Gaussian quadrature with a relatively modest number of points. The presence of bound states between $\mu_{\min }$ and $\mu_{\max }$ is actually problematic, giving rise to singularities in $G^{<}$ which manifest themselves through convergence problems. Fortunately, most of the systems investigated to date are free from this problem, and so the integration of the second term of Eq. (9) is straightforward. The exceptions here are some of the carbon nanotube systems considered in this paper. The explicit handling of the bound states is somewhat subtle, and requires both a symmetry decomposition of the charge density (discussed in Sec. III), and a shifting of the integration limits (described in Sec. IV).

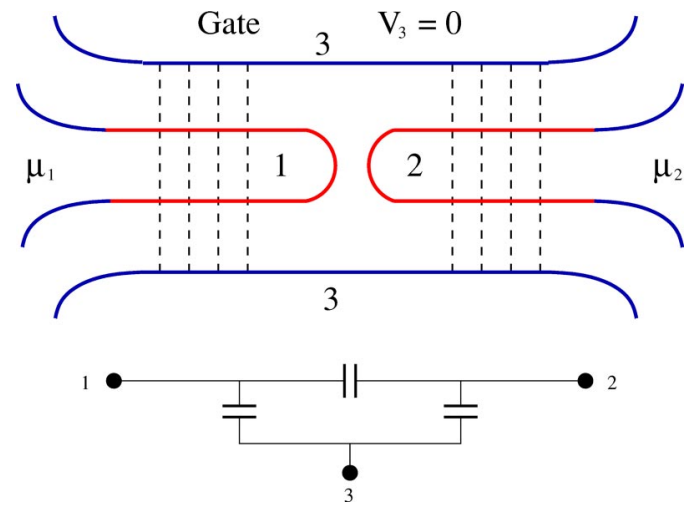

FIG. 2. (Color online) Schematic of the device used to calculate capacitance for a generic two-probe system. Note that the system is surrounded by a metal gate to keep in the field lines. The gate potential $V_{3}=0$ sets the energy and electrochemical scale for the system. The equivalent circuit of the system is shown below.

Having computed the charge density, it is straightforward to calculate the linear capacitance coefficients, hereafter simply referred to as the capacitance coefficients, using the definition $C_{\alpha \beta}=e d Q_{\alpha} / d \mu_{\beta}$; i.e., by applying a change in voltage $\Delta V=d \mu / e$ to a given reservoir and then measuring the charge difference $\Delta Q=Q(V+\Delta V)-Q(V)$. As a further feature, we have used the Dirichlet boundary conditions for the electrostatic potential at the walls of our finite-sized calculational box, which corresponds to the entire system being surrounded by a metal container, ${ }^{33}$ as shown in Fig. 2. This box is needed in order to terminate any field lines that emanate from the system. In the limit of an infinite-sized container, the computed results reduce to that of a nanotube system in free space. A further advantage is that it allows for the treatment of charged nanotube systems. However, the nanotubes do interact with walls of the container, so that the equivalent circuit of the system is characterized by three capacitors, as illustrated in Fig. 2. As will be discussed, this primarily leads to self-charging effects. Finally, since we are mostly dealing with two-conductor systems, the main quantities calculated are

$$
\begin{aligned}
& \Delta Q_{1}=C_{11} \Delta V_{1}+C_{12} \Delta V_{2}, \\
& \Delta Q_{2}=C_{21} \Delta V_{1}+C_{22} \Delta V_{2} .
\end{aligned}
$$

Here, the $C_{\alpha \alpha}$ matrix coefficients represent the "selfcharging" and $C_{\alpha \beta}$ the "mutual-charging" terms of the conductors.

\section{SYMMETRY ANALYSIS OF NANOTUBE CHARGE DENSITY}

Because carbon nanotubes are structures with a high degree of symmetry, there is opportunity for significantly reducing the computational costs of the calculations by means of a group theory analysis. ${ }^{34}$ This is important because most of the nanotube systems we consider consist of several hundreds of atoms, and are therefore computationally quite expensive. These savings are accomplished by means of recasting the problem with a set of hybrid molecular orbitals, 
which are obtained in terms of the irreducible representation of the symmetry group of the nanotube. This approach has the further advantage of allowing one to classify the chargedensity contributions in terms of the different symmetry properties of the wave functions. This not only gives insight into the induced charge, but is also important for technical reasons in order to properly deal with the bound states present in the system.

Here, we briefly outline our method for constructing such a symmetric basis. For brevity, only the highly symmetric armchair and zigzag tubes will be considered. These are described by symmorphic groups, for which the translations and rotations are decoupled from each other so that rotations can be treated as point-group operations. For infinitely long $(n, n)$ armchair, or $(n, 0)$ zigzag tubes, the symmetry group is $D_{n h}$ for even $n$ and $D_{n d}$ for odd $n$. For our purposes, it is sufficient to use the lower point rotation group $C_{n}$, which is a subgroup of both $D_{n h}$ and $D_{n d}$. The $C_{n}$ group consists of $n$ rotation operations, through angles $\{0,2 \pi / n, 2(2 \pi / n), \ldots,(n-1) 2 \pi / n\}$, with the axis of rotation running along the center of the nanotube. We define a symbol $\mathbf{C}_{\mathbf{n}}$ to denote rotation through angle $2 \pi / n$. In this notation, the group $C_{n}$ can be thought to consist of operations $\left\{\mathbf{C}_{n}^{0}, \mathbf{C}_{\mathbf{n}}^{1}, \ldots, \mathbf{C}_{\mathbf{n}}^{n-1}\right\}$, where the superscript indicates the number of times $\mathbf{C}_{\mathbf{n}}$ is applied successively, with $n=0$ corresponding to the identity element of the group. The parent groups $D_{n h}$ and $D_{n d}$ contain, in addition to the elements of $C_{n}, n \mathbf{C}_{2}$ rotation axes plus additional plane reflection symmetries (one horizontal plane for $D_{n h}$ and $n$ dihedral planes for $\left.D_{n d}\right)$. The $C_{n}$ symmetry also holds for a perfect open semi-infinite $(n, n)$ or $(n, 0)$ tube. For some half-tubes, it is also possible to construct a cap at the end of the tube which shares the $C_{n}$ symmetry and, whenever possible, we will make use of such structures. Finally, for a junction between $(n, n)$ and $(n, 0)$ tubes, $C_{n / 2}$ symmetry will hold if the center axes of the two tubes coincide.

Consider a nanotube (or a junction of semi-infinite nanotubes) with rotational symmetry $N$. For such tubes, any rotation of $C_{N}$ will take a given atom from its site to another atomic site. When the atom is moved by rotation, its associated Cartesian wave functions are rotated with it. When the rotation is completed, it is possible to express the rotated wave functions in terms of the unrotated wave functions at the new atomic site. Hence one can think of each rotation as taking an orbital and expressing it as a combination of new orbitals at the new atomic site. Thus the wave functions $\Psi$ before and $\Psi^{\prime}$ after rotation operation $C_{N}$, both expressed in terms of the Cartesian basis, are related through a rotation operator $\mathbf{O}_{C_{N}}$ such that

$$
\mathbf{O}_{C_{N}} \Psi=\Psi^{\prime} .
$$

As the system is described by symmetry group $C_{n}$, the Hamiltonian $H$ and the overlap matrix $S$ of the system must also be consistent with the symmetry group. In particular, expectation values must obey $\langle\Psi|H| \Psi\rangle=\left\langle\Psi^{\prime}|H| \Psi^{\prime}\right\rangle$, since applying a rotation $C_{N}$ must leave the system properties invariant. It therefore follows straightforwardly that the following commutation relations must hold:
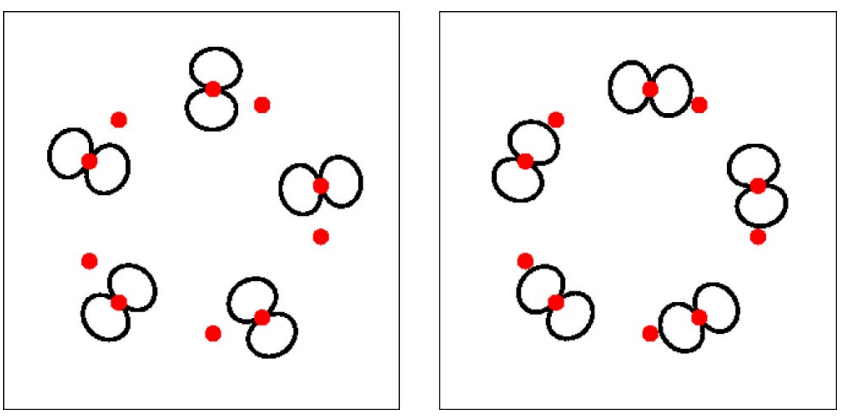

FIG. 3. (Color online) Schematic of $\Sigma p_{y^{\prime}}$ and $\Sigma p_{x^{\prime}}$ symmetric orbitals constructed on a ring of $(5,5)$ tube out of $p_{x}, p_{y}$ orbitals.

$$
\begin{gathered}
\mathbf{O}_{C_{N}} H=H \mathbf{O}_{C_{N}}, \\
\mathbf{O}_{C_{N}} S=S \mathbf{O}_{C_{N}} .
\end{gathered}
$$

The Cartesian basis orbitals $\left\{\phi_{\mu}\right\}$ form a basis for a reducible representation of $C_{N}$. We need to find combinations of $\left\{\phi_{\mu}\right\}$ which will serve as basis for the irreducible representation of $C_{N}$. Such a basis may be constructed in two stages. First, it is necessary to change the axis of the Cartesian orbitals from the global coordinates of the threedimensional $\{x, y, z\}$ grid to atom specific axes which are more appropriate for the treatment of the nanotubes. Starting from the Cartesian basis orbitals $\left\{\phi_{s}, \phi_{p_{x}}, \phi_{p_{y}}, \phi_{p_{z}}\right\}$, we construct tube frame orbitals $\left\{\phi_{s}^{\text {tube }}, \boldsymbol{\phi}_{p_{x^{\prime}}}^{\text {tube }}, \boldsymbol{\phi}_{p_{y^{\prime}}}^{\text {tube }}, \phi_{p_{z^{\prime}}}^{\text {tube }}\right\}$ where the $y^{\prime}$ axis runs along the direction from tube center to the atom, $x^{\prime}$ is tangent to tube circumference, and $z^{\prime}$ is along the longitudinal direction of the tube (and hence unchanged), as shown in Fig. 3. Hence, for each atom, we can use the new orbital basis defined as

$$
\begin{gathered}
\phi_{s}^{\text {tube }}=\phi_{s}, \\
\phi_{p_{x^{\prime}}}^{\text {tube }}=\cos (\theta) \phi_{p_{x}}+\sin (\theta) \phi_{p_{y}}, \\
\phi_{p_{y^{\prime}}}^{\text {tube }}=-\sin (\theta) \phi_{p_{x}}+\cos (\theta) \phi_{p_{y^{\prime}}}, \\
\phi_{p_{z^{\prime}}}^{\text {tube }}=\phi_{p_{z^{\prime}}},
\end{gathered}
$$

where $\theta$ is the angle between $y$ and $y^{\prime}$ axes specifying the angular orientation of the atom around the central axis of the nanotube. We now proceed to construct new symmetry orbitals $\phi^{\text {sym }}$ from combinations of $\phi^{\text {tube }}$. We recall that our system is described by symmetry group $C_{N}$. In such a system atoms are arranged in sets of rings, located in a series along the longitudinal direction of the tube, where ring $r$ contains $K_{r}$ atoms and obeys symmetry $C_{N}$, so that $K_{r}$ is divisible by $N$. To illustrate, we have $K_{r}=2 n$ for a $(n, n)$ tube and $K_{r}=n$ for a $(n, 0)$ tube. We divide $\phi^{\text {tube }}$ orbitals into subsets containing $4 K_{r}$ orbitals belonging to atoms of ring $r$, so that

$$
\phi_{\mu^{\prime}, r, I^{\prime}}^{\text {tube }} \equiv \phi_{\mu^{\prime}, I}^{\text {tube }},
$$

where atom $I$ is reindexed as atom $I^{\prime}$ belonging to ring $r$ and $\mu=\left\{s, p_{x}, p_{y}, p_{z}\right\}$. As each ring has $N$ rotational symmetry, 
we can further divide the orbitals on ring $r$ into $N$ subsets of $4 K_{r} / N$ orbitals which will transform into each other under rotation operations of $C_{N}$. Thus, the final indices are such that for a rotation through angle $2 \pi / N$,

$$
\mathbf{O}_{C_{N}} \phi_{\mu^{\prime}, r, p, m}^{\text {tube }} \equiv \phi_{\mu^{\prime}, r, p,[\bmod (m+1, N)]}^{\text {tube }},
$$

where $p$ indexes the subset of orbitals that map into each other and $m$ is the orbital index within this subset, which denotes the angular ordering of its atoms along the ring. Because of the previous transformation to the tube frame, each subset $p$ contains orbitals of same $\mu$ which get rotated into each other under action of elements of $C_{N}$. Finally, using this scheme, normalized symmetry orbitals are obtained as

$$
\phi_{\nu, l}^{s y m}=\phi_{\mu^{\prime}, r, p, l}^{s y m}=\frac{1}{\sqrt{N}} \sum_{m=1}^{N} \phi_{\mu^{\prime}, r, p, m} \epsilon^{m l},
$$

where $l=\{0, \pm 1, \ldots, \pm(N-1) / 2\} \quad$ for $N$ odd, $l=\{0$, $\pm 1, \ldots, \pm[(N / 2)-1],+N / 2\}$ for $N$ even, and $\epsilon=e^{i 2 \pi / N}$. For ease of notation, we have also defined a new collective index $\nu$, which from now on refers to all the indices except the $l$ associated with the symmetry, i.e., $\nu=\left\{\mu^{\prime}, r, p\right\}$.

Given the way we defined it, the symmetric state has the property that under $C_{N}$ the state transforms into itself multiplied by a constant:

$$
\mathbf{O}_{C_{N}} \phi_{\nu, l}^{s y m}=\epsilon^{l} \phi_{\nu, l}^{s y m} .
$$

In group theory this property means that symmetry orbitals with different $l$ form a basis for different irreducible representations of $C_{N}$. It is easy to see that any matrix elements of Hamiltonian matrix $H$ (and overlap matrix $S$ ) between symmetry orbitals from different representations must vanish. Using the behavior of the symmetric states under $\mathbf{O}_{C_{N}}$ and recalling that $\mathbf{O}_{C_{N}}$ commutes with $H$ [see Eq. (12)], we note

$$
\begin{aligned}
\left\langle\phi_{\nu^{\prime}, l^{\prime}}^{\text {sym }}|H| \phi_{\nu, l}^{\text {sym }}\right\rangle & =\left\langle\phi_{\nu^{\prime}, l^{\prime}}^{\text {sym }}\left|\mathbf{O}_{C_{N}}^{-1} \mathbf{O}_{C_{N}} H \mathbf{O}_{C_{N}}^{-1} \mathbf{O}_{C_{N}}\right| \phi_{\nu, l}^{s y m}\right\rangle \\
& =\epsilon^{l-l^{\prime}}\left\langle\phi_{\nu^{\prime}, l^{\prime}}^{s y m}|H| \phi_{\nu, l}^{s y m}\right\rangle .
\end{aligned}
$$

This implies that the matrix element must vanish unless $l$ $=l^{\prime}$. In other words, $H$ and $S$ matrices become block matrices-a property that is very useful for the speeding up of the numerical calculations.

In many cases, it is advantageous to work with a real basis. Such a real symmetric basis set $\left\{\phi_{\nu, L}^{s y m}\right\}$ may be formed from combinations of the basis functions $\left\{\phi_{\nu, l}^{\text {sym }}\right\}$ by means of

$$
\begin{gathered}
\phi_{\nu, L}^{s y m}=\phi_{\nu, l}^{s y m}, \quad L=l, 0 ; \\
\phi_{\nu, L}^{s y m}=\left\{\begin{array}{l}
\frac{1}{\sqrt{2}}\left(\phi_{\nu, l}^{s y m}+\phi_{\nu,-l}^{s y m}\right) \\
\frac{i}{\sqrt{2}}\left(\phi_{\nu, l}^{s y m}-\phi_{\nu,-l}^{s y m}\right)
\end{array}\left(L=|l| ; 1 \leqslant|l| \leqslant \frac{N-1}{2}\right),\right.
\end{gathered}
$$

$$
\phi_{\nu, L}^{s y m}=\phi_{\nu, l}^{s y m}, \quad L=l ; L=\frac{N}{2} \quad \text { if } N \text { even. }
$$

It clearly follows that for the real basis, the matrix elements $\left\langle\phi_{\nu^{\prime}, L^{\prime}}^{\text {sym }}|H| \phi_{\nu, L}^{\text {sym }}\right\rangle$ must vanish unless $L=L^{\prime}$.

In order to take advantage of the fact that large blocks of elements of the $H$ and $S$ vanish in the new, symmetric basis, we must rewrite all the relevant quantities in this basis. Specifically, let us briefly consider converting Hamiltonian matrix $H_{A B}^{c a r}$, defined in the Cartesian basis, between two (arbitrary) spatial regions labeled $A$ or $B$. Matrices for a change of basis may be written in the standard way as

$$
\begin{gathered}
T_{A, \nu l, I}=\left(\phi_{\nu l}^{s y m}\right)_{I}, \\
T_{B, \nu^{\prime} l^{\prime}, I^{\prime}}=\left(\phi_{\nu^{\prime} l^{\prime}}^{s y m}\right)_{I^{\prime}} .
\end{gathered}
$$

These matrices carry along indices for the region that is transformed ( $A$ or $B$ ), the index for the symmetric basis, and the index for the Cartesian basis. Each column of these matrices represents one of the symmetric states expressed in terms of the nonsymmetric Cartesian basis. We apply the change of basis matrix to obtain $H$ in the symmetric basis:

$$
H_{A B}^{s y m}=\left(T_{A}\right)^{-1} H_{A B}^{c a r} T_{B} .
$$

The elements of this matrix are just

$$
H_{A B, \nu l, \nu^{\prime} l^{\prime}}^{s y m}=\left\langle\phi_{\nu l}\left|H_{A B}\right| \phi_{\nu^{\prime} l^{\prime}}\right\rangle,
$$

which will vanish if $l \neq l^{\prime}$ and, hence, the matrix $H_{A B}^{s y m}$ is a block matrix as well.

We now describe how to take advantage of the block nature of the transformed matrices. Here, we shall only discuss in detail two-probe systems. Use of the real, symmetric basis as defined by Eq. (19) ensures that $H, S$, and $\hat{\rho}$ are real block matrices, which reduces the memory storage requirements. Now each family of nonzero submatrices associated with index $L$ can be treated as an independent problem, to which the methodology outlined in the preceding section may be applied. However, now the nonzero block matrices will be reduced in size by a factor of $2 / N$ relative to the full matrices, except for the $L=0, N / 2$ cases which are reduced by a factor of $1 / N$ when compared to the complex symmetric basis. Hence, one obtains a family of $N / 2$ equations for $N$ even and $(N+1) / 2$ for $N$ odd. Quantities such as the block matrix for the retarded Green's function, indexed by $L$, are then given by

$$
\widetilde{G}^{R, s y m, L}=\left[\widetilde{H}^{s y m, L}-E \widetilde{S}^{s y m, L}-\widetilde{\Sigma}^{s y m, L}\right]^{-1},
$$

with $\widetilde{\Sigma^{s y m, L}}$ representing the block matrix for the sum of the self-energies from the left and right leads. With block matrix $\widetilde{G}^{R, s y m, L}$ the density of states (DOS) of the scattering region can now be expressed as

$$
\frac{d n}{d E}=\sum_{L} \operatorname{Im}\left[\operatorname{Tr}_{C}\left(\widetilde{S}_{C C}^{s y m, L} \widetilde{G}_{C C}^{s y m, L}\right)\right]=\sum_{L} \frac{d n_{L}}{d E},
$$




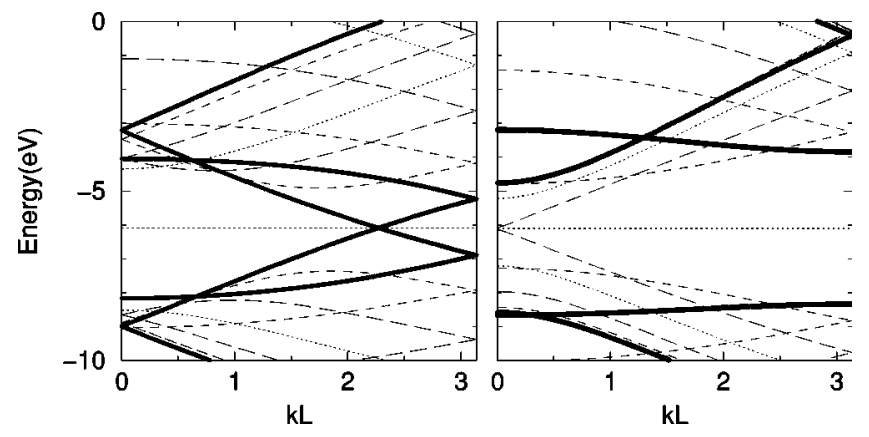

FIG. 4. Symmetry decomposed bands of $(12,0)$ and $(6,6)$ tube: $L=0$ (solid line), $L=1$ (dashed line), $L=2$ (long dashed line), and $L=3$ (dotted line)

which decomposes the DOS into its component contributions from wave functions having a different symmetry $L$. Similarly, one computes

$$
\widetilde{G}^{<, s y m, L}=\widetilde{G}^{R, s y m, L} \widetilde{\Sigma}^{<, s y m, L} \widetilde{G}^{A, s y m, L},
$$

with the Fermi occupation function defined as before, to obtain the submatrix of the full symmetric density matrix $\rho^{s y m}$ :

$$
\begin{aligned}
\tilde{\rho}^{s y m, L}= & \frac{2}{\pi} \operatorname{Im}\left[\int_{-\infty}^{\min \left(\mu_{l}+V_{l}, \mu_{r}+V_{r}\right)} d E \widetilde{G}^{R, s y m, L}(E)\right] \\
& +\frac{1}{\pi} \operatorname{Re}\left[\int_{\min \left(\mu_{l}+V_{l}, \mu_{r}+V_{r}\right)}^{\max \left(\mu_{l}+V_{l}, \mu_{r}+V_{r}\right)} d E \widetilde{G}^{<, s y m, L}(E)\right] .
\end{aligned}
$$

Generally, the limits of the energy integrals and integration contours are the same as before. However, as already noted, adjustments may need to be made in the presence of bound states. By expanding the submatrices $\tilde{\rho}^{s y m, L}$ into the fullsized matrices $\hat{\rho}^{\text {sym,L}}$, and using the appropriate change of basis matrix $T$, one obtains the expression for the total density matrix

$$
\hat{\rho}=\sum_{L} \hat{\rho}^{L}=\sum_{L} T^{\dagger} \hat{\rho}^{s y m, L} T
$$

which is now decomposed into contributions from each symmetry index $L$. Again, the method vastly speeds up calculation as the time consuming steps of determining $\Sigma, G^{R}$, and $G^{<}$generally speed up by factor $O\left((N / 2)^{3}\right) /(N / 2)$ $=O\left((N / 2)^{2}\right)$.

A very similar approach can be used for the calculation of the density matrix and symmetry analysis for a periodic system. As an example of this, we have applied the symmetry decomposition to the nanotube band structures, as shown in Fig. 4 for the $(12,0)$ and $(6,6)$ nanotubes. Note that near the Fermi level, the $L=0$ and $L=2$ bands dominate the $(12,0)$ and $(6,6)$ nanotube system, respectively. This analysis is important for the discussion of the $(12,0) /(6,6)$ metal-metal nanotube junction in Sec. IV C.

In summary, in this section we have outlined a way of rewriting the problem in terms of new set of hybrid orbitals determined by the irreducible elements of the rotational group for the specific carbon nanotube under consideration. This decomposes the problem into different, independent block matrices, which are labeled by $L$ (the symmetry index of the wave functions), and has the advantage of significantly speeding up the code.

\section{CAPACITANCE OF CARBON NANOTUBE SYSTEMS}

Having discussed the methodology and the symmetry decomposition of the charge density, we present our capacitance calculations for prototypical carbon nanotube systems. Specifically, we have investigated the capacitance of nested two- and three-shelled nanotubes, the insertion of one nanotube into another, a nanotube junction, and use of a nanotube as a capacitance probe over a flat $\mathrm{Al}$ surface. ${ }^{35}$ We have limited our studies to system in which there is no direct current (dc) flowing between any of the conductors, which means that there is negligible overlap between the electronic wave functions of the different conductors.

\section{A. Multiwall tubes in a periodic geometry}

For the first example, we consider the capacitance per unit length of two nested armchair nanotubes. Such systems, which corresponds to that of two multiwalled nanotube shells, has recently been realized experimentally. ${ }^{36,37}$ Specifically, we looked at the case of a metallic $(5,5)$ nanotube (conductor 1$)$, inside a larger $(m, m)$ nanotube (conductor 2$)$, with helicity index $m$ ranging from 12 to 22 . For the smallest $(12,12)$ tube, the closest distance between atoms on the different tubes is $9.1 \AA$. The atomic cutoff radius used was 4.7 $\AA$, so that all the tubes are de facto separate entities, except for presence of the real-space electrostatic potential which couples the two tubes. By connecting these tubes to two different reservoirs, a quantum system that is analogous to a classical, concentric cylindrical capacitor is constructed.

The simulations were carried out in a periodic configuration, with one unit cell of a $(5,5)$ tube with 40 carbon atoms, and one unit cell of the larger $(n, n)$ tubes with $8 n$ carbon atoms. Here, the use of the symmetry analysis turned out to be important as we were able to study systems up to $n$ $=22$, which contains 216 atoms in the unit cell. Central simulation box sizes of $40 \times 40 \times 4.8 \AA^{3}$ discretized on a grid of $256 \times 256 \times 32$ and $80 \times 80 \times 4.8 \AA^{3}$ on a grid of 512 $\times 512 \times 32$ were used. Each calculation used $200 k$ points in our sampling.

The electrochemical potential of the system is measured with respect to an arbitrary reference potential, which we set as follows. First, by imposing a $U(r)=V_{\text {gate }}$ in the longitudinal direction on the walls of the container surrounding the nanotube system, we are able to impose $\mu_{1}$ and $\mu_{2}$ on each of the two tubes. These are set by changing the external bias voltage on the reservoirs coupled to the tubes. We set $\mu$ by fixing $V_{\text {gate }}=0$, and then finding $\mu_{1}=\mu_{2}=\mu_{o}$ such that the two-tube system is neutral. This also determines the origin of our energy scale. The capacitance matrix coefficients are then determined as with respect to $\mu_{o}$, as described in Sec. II. For example, if potential $\mu_{1} \rightarrow \mu_{1}+d \mu_{1}$, then the first tube acquires charge $d Q_{1}$, and the second tube acquires 

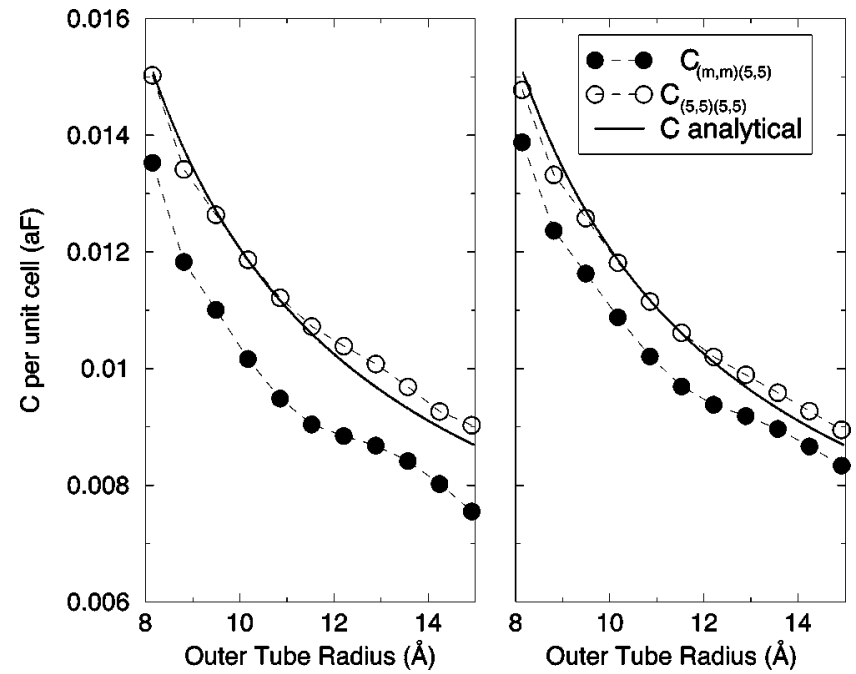

FIG. 5. Capacitance vs tube radius for $(5,5)$ shell inside $(m, m)$ tubes. The left (right) panel shows data for a $40 \times 40 \AA^{2}(80$ $\times 80 \AA^{2}$ ) metal container enclosing the system. The analytical results are obtained as described in the text and the Appendix, and are marked with a solid line.

charge of the opposite sign $d Q_{2}$ in response. Both $C_{11}$ and $C_{12}$ are then easily determined. Similarly, $C_{21}$ and $C_{22}$ are found by changing $\mu_{2}$. In discussing the capacitance results, it will be convenient to label the capacitance coefficients with $(n, m)$ helicity indices of the nanotubes. For instance, $C_{(12,12),(5,5)}$ gives the charge accumulation on the $(12,12)$ tube in response to a variation of the electrochemical potential of the $(5,5)$ tube reservoir.

First, we consider the case of a $(5,5) /(12,12)$ nanotube system, all in a metal container $40 \times 40 \AA^{2}$ in the plane perpendicular to the nanotube axis. The capacitance response is essential linear, to better than $1 \%$, to applied voltages of at least $5 \mathrm{~V}$, and so that well-defined voltage-independent capacitance coefficients are readily obtained. For the $(5,5) /$ $(12,12)$ system, capacitance coefficients per unit cell of

$$
\begin{gathered}
C_{1,1}=C_{(5,5)(5,5)}=0.0150 \mathrm{aF}, \\
C_{2,1}=C_{(12,12)(5,5)}=-0.0135 \mathrm{aF}, \\
C_{1,2}=C_{(5,5)(12,12)}=-0.0134 \mathrm{aF}, \\
C_{2,2}=C_{(12,12)(12,12)}=0.0243 \mathrm{aF}
\end{gathered}
$$

were obtained $\left(1 \mathrm{aF}=10^{-18} \mathrm{~F}\right)$. If the system consisted of two nested nanotubes in free space, the magnitude of all four coefficients would be the same. However, since we are dealing with a finite system surrounded by a metal box, the outer nanotube will interact with this box, as schematically illustrated in Fig. 2. Thus, the $C_{(12,12)(12,12)}$ coefficient is expected to be larger in magnitude. In response to the potential variation at $(12,12)$ tube, the induced charge on $(5,5)$ amounts to only $55 \%$ of the charge on $(12,12)$, indicating that the rest of the induced charge is on the container. In contrast, if charge is injected into the $(5,5)$ tube, then the $(12,12)$ tube does most of the screening, with $89 \%$ of the induced charge found on the $(12,12)$ tube. We also find that $C_{(12,12)(5,5)}$ and
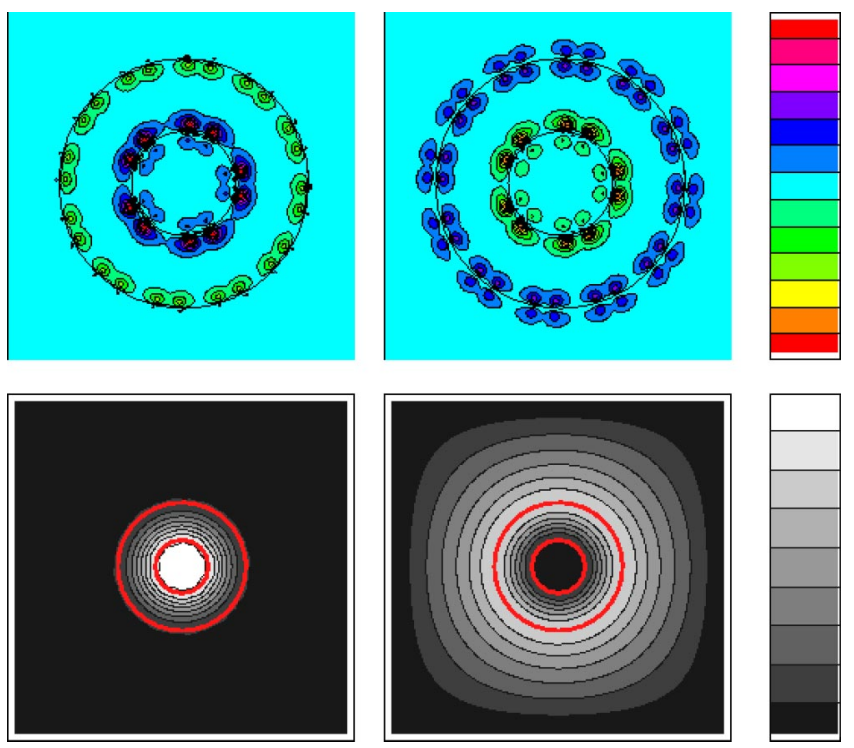

FIG. 6. (Color online) The charge-density variation $d Q$ (upper panels) and characteristic potentials (lower panels) for the nested $(5,5) /(12,12)$ system. The electrochemical potential variation is applied to the inner $(5,5)$ tube (left panels) and the outer $(12,12)$ tubes (right panels). From these plots, $u \approx 0.9$ just inside the inner tube and $u \approx 0.75$ just inside the outer tube.

$C_{(5,5)(12,12)}$ agree with each other to better than $1 \%$, which is an important check on the quality of our calculated results. Since the charge injected into the $(5,5)$ tube is almost completely screened by the response charge of the $(12,12)$ tube, one can expect that the $C_{(5,5),(5,5)}$ will change only slightly as the container size is increased. That is indeed what is observed, as shown in Fig. 5. As the metal container gets larger, the outer tube screens more of the charge on the inner tube, and so the numerical values of the two coefficients become closer and closer. We estimate that in the infinite container limit, all $(5,5) /(12,12)$ capacitance coefficients will have a numerical magnitude of $0.015 \mathrm{aF}$.

Figure 6 shows the bias-induced variation in the charge density and in the scaled electrostatic potential $u$ $=e d U(r) / d \mu$. From the induced charge-density plots, it is clear that the nanotubes polarize, at least to some extent. The polarization of the outer tube is much less when the electrochemical potential is changed there, because a significant portion of the induced charge will be found on the metal gate surrounding the system. Note that when the charge is injected into the inner tube, most of the characteristic potential is confined to the space between the two nanotubes, which is not the case when the outer tube is charged. By means of this characteristic potential, the charging mechanism may easily be explained. If $d \mu_{1}$ is applied to the first tube, the Fermi energy shifts by $d \mu_{1}$ and the band structure responds by shifting up by $u_{1} d \mu_{1}$, where $u_{1}(r)$ is averaged over the volume of the tube. However, $u_{1}<1$ inside the first tube, and hence the tube acquires a net charge $(1-u)\left(d n_{1} / d E\right) d \mu_{1}$, using the DOS of the tube averaged over the appropriate energy window. In the second tube, the electrochemical potential stays the same, while the bands are shifted upwards by $u_{1} d \mu_{1}$ [using $u_{1}(r)$ averaged over tube 2]. Hence some bands empty out and the second tube acquires a net charge $-u\left(d n_{2} / d E\right) d \mu$. 
Estimating the value of $u$ from the plot of the characteristic potential (Fig. 6), we see that the tube band structure will shift relative to the Fermi energy by only $\sim 0.1 d \mu$. Within maximum applied bias of $5 \mathrm{~V}$, this will not be sufficient to shift the Fermi energy of the $(5,5)$ nanotube into the energy region with a nonlinear DOS (recall that the armchair tubes have linear bands over a large region about the Fermi level). Therefore, the capacitance is also expected to be constant in this voltage range, which is what we observe.

To gain further insight into the relation between the classical and quantum capacitance coefficients, we have derived a simple analytical formula based on the Büttiker capacitance formalism, ${ }^{3}$ outlined in the Appendix. The relevant system geometry here consists of two thin, concentric cylindrical tubes of length $l$, which are treated as a twodimensional electron gas. It is assumed that these tubes do not interact with the boundary container, and that the system is always neutral. All capacitance coefficients will therefore have equal magnitude $C$, with a value of

$$
\frac{2 \pi \epsilon_{o} l}{C}=\ln \left(\frac{R_{2}}{R_{1}}\right)+\left(\frac{\lambda_{1}}{R_{1}}\right)+\left(\frac{\lambda_{2}}{R_{2}}\right) .
$$

Here $R_{1,2}$ are the radii of the outer and inner tube, respectively, $\lambda^{-1}=4 \pi e^{2}(d \sigma / d E)$ is the screening length, and $d \sigma / d E$ is the density of states per unit area of the tube cylinder. These parameters were all estimated from the $a b$ initio data. The factors $\lambda_{1} / R_{1}$ and $\lambda_{2} / R_{2}$ all have a constant value of about 0.15 for the armchair tubes, since the DOS at that Fermi energy is approximately constant. We estimate the geometric parameters $R_{1,2}$ as the average radius of the charge distribution of each nanotube, and these take on values of $(8.14-0.54) \AA$ for a $(12,12)$ and $(3.39+0.73) \AA$ for the $(5,5)$ tubes, respectively. With these parameters, we have calculated all the capacitive coefficients and plotted the results in Fig. 5. Note that the agreement between this simple model and the $a b$ initio capacitive coefficients is quite good. Also, for the nested nanotube system, the quantum corrections $\lambda / R$ are quite significant and comparable to the classical logarithmic term. The classical limit, which is reached when the first term of Eq. (29) is much larger than the screening terms, which holds for $R_{1} \gg 1.35 R_{2}$, which is a limit that is not reached for our largest tubes.

As a further test of the nanoscale capacitance, we have considered the case of three nested nanotubes. Specifically, the three shells considered consisted of $(36,0),(22,0)$, and $(9,0)$ tubes, so that the innermost and outermost tubes are both metallic and separated from each other by a semiconducting tube. In analogy to the classical case, one expects that the semiconducting tube now acts as a dielectric, and will therefore increase the value of the capacitance. Since the intermediate $(22,0)$ tube does not acquire substantial charge when a voltage is applied to its reservoir, we consider the capacitance coefficients between the $(36,0)$ and $(9,0)$ tubes only. In the absence of any intermediate semiconducting nanotube, the capacitance coefficients are

$$
\begin{gathered}
C_{(9,0),(9,0)}=0.0164 \mathrm{aF}, \\
C_{(36,0),(9,0)}=-0.0117 \mathrm{aF} .
\end{gathered}
$$

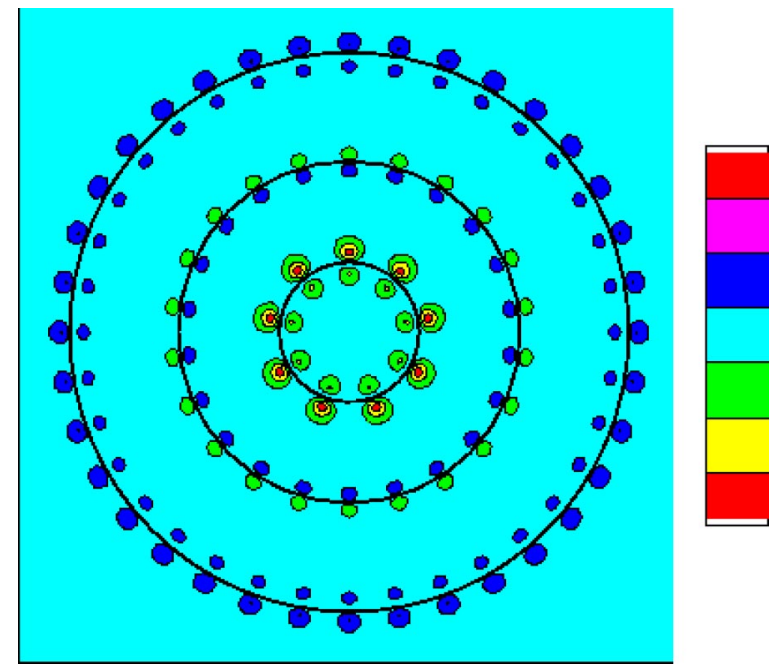

FIG. 7. (Color online) Charge-density variation for the case of three nested nanotubes. Here, the bias voltage has been applied to the outermost $(36,0)$ tube. Note the polarization response in the form of induced dipoles on the intermediate $(22,0)$ semiconducting tube.

In the presence of an intermediate semiconducting tube, the capacitance coefficients are

$$
\begin{gathered}
C_{(9,0),(9,0)}=0.0180 \mathrm{aF}, \\
C_{(36,0),(9,0)}=-0.0135 \mathrm{aF}, \\
C_{(9,0),(36,0)}=-0.0137 \mathrm{aF}, \\
C_{(36,0),(36,0)}=0.0494 \mathrm{aF} .
\end{gathered}
$$

Clearly, the presence of the semiconducting tube enhances the value of the cross capacitance by about $10 \%$. We can see precisely how this comes about by examining the change in charge density, as shown in Fig. 7. Clearly, even though the semiconducting tube does not acquire any net charge, its electrons exhibit a polarization response such that a small dipole is induced about each carbon atom in response to the electric field due to the applied voltage. This increases the value of the capacitance in analogy to the classical case. ${ }^{38}$ Further increasing the number of semiconducting nanotube shells between the two metal nanotubes is therefore expected to boost the numerical values of the capacitance coefficients considerably.

\section{B. Two-probe capacitance}

We now turn to the case of a two-probe system, in which a capped $(5,5)$ nanotube is inserted a finite distance into an open $(12,12)$ nanotube, with the central axis of the two tubes coinciding, as shown in Fig. 8. The system now consists of two semi-infinite carbon nanotube leads, and a central region containing the junction. All in all, the system consisted of 458 carbon atoms in a $40 \times 40 \times 34 \AA^{3}$ to $40 \times 40 \times 64 \AA^{3}$ box, discretized on a $256 \times 256 \times 512$ grid. For our calculations, we choose to measure the electrochemical potential with respect to a state where the two nanotubes leads, en- 


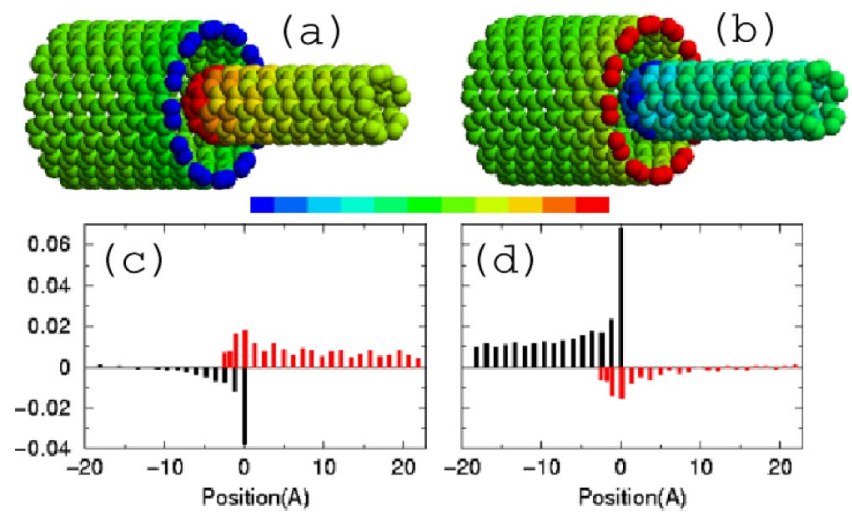

FIG. 8. (Color online) Charging of $(12,12) /(5,5)$ tube junction as $(5,5)$ tube is inserted into the open $(12,12)$ tube, for a central simulation box with 458 atoms. A $+0.272 \mathrm{eV}$ bias is applied to the right $(5,5)$ tube in $(a, c)$, and on the left $(12,12)$ tube in $(b, d)$. Upper panel show gray-scale plots of charge accumulation with $(5,5)[(12,12)]$ tube on the left (right) corresponding to charge addition and $(12,12)$ $[(5,5)]$ tube subject to charge depletion. The lower panels display histogram plots of the charge accumulated on the tube rings, corresponding to the geometry shown in $(\mathrm{a}, \mathrm{c})$.

closed in a box with $V_{\text {gate }}=0$, are as close to neutrality as possible. The electrochemical potential for a neutral $(12,12)$ tube is found to be $-6.127 \mathrm{eV}$ and $-6.071 \mathrm{eV}$ for the neutral $(5,5)$ tube. These potentials are very close, and we set the reference zero-bias potential to be halfway between these two values. This implies a net gain of 0.0042 electrons per unit for the $(12,12)$ tube, and a loss of 0.0025 electrons per unit cell from the $(5,5)$ tube. Clearly, these extra charges are so small that the nanotube system deviates only very slightly from perfect neutrality. As with the other calculation, the charge-voltage characteristics are essentially linear within $1 \%$, at least up to a bias of $1 \mathrm{~V}$.

The charging of the tube is shown in Fig. 8, which also shows both a gray-scale plot of the charge variation and a more quantitative measure in the form of a histogram plot of the change in the total charge accumulated on each nanotube ring. We note that the $(12,12)$ tube acquires a very large amount of charge on its terminal ring, which is most likely due to the presence of its dangling bonds. For the particular configuration shown, the capacitance coefficients are

$$
\begin{gathered}
C_{(5,5)(5,5)}=0.1050 \mathrm{aF}, \\
C_{(12,12)(5,5)}=-0.0455 \mathrm{aF}, \\
C_{(5,5)(12,12)}=-0.0451 \mathrm{aF}, \\
C_{(12,12)(12,12)}=0.1565 \mathrm{aF} .
\end{gathered}
$$

Again the capacitance coefficients $C_{(5,5)(12,12)}$ and $C_{(12,12)(5,5)}$ are seen to agree to within $1 \%$. Note that nanotube, to which the bias is applied, gains charge along its entire length within the junction region. This self-charging is due to the capacitive coupling between the nanotube and the surrounding box. Clearly, the values of the $C_{\alpha \alpha}$ terms will increase linearly in size as more and more of the charge density is included. As the size of the container surrounding the system is increased,

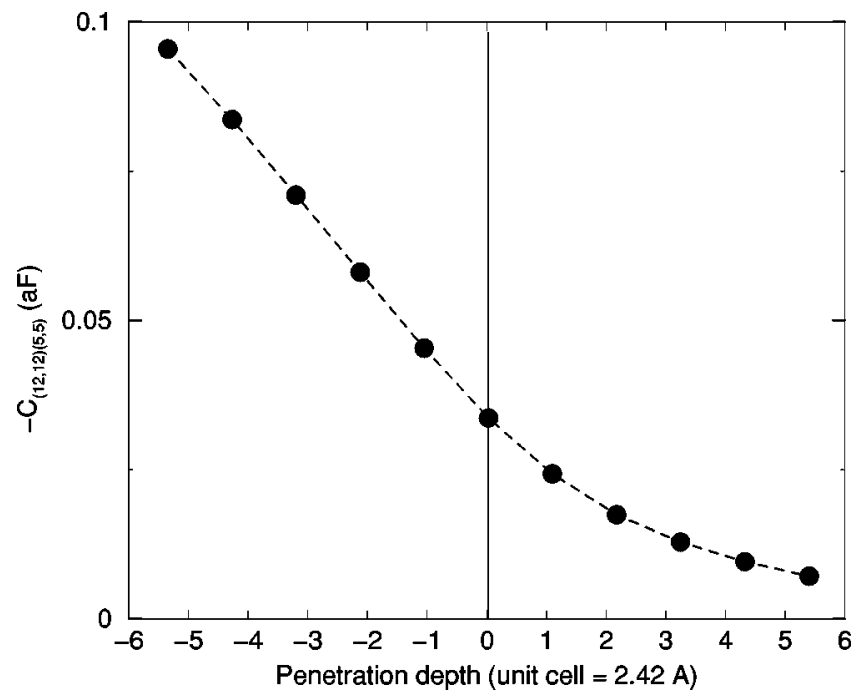

FIG. 9. Capacitance $\left(C_{(12,12)(5,5)}\right)$ vs penetration depth for capped $(5,5)$ inside $(12,12)$ tube.

the capacitance coupling between the container and lead tubes will progressively decrease, and the charge accumulated on the tube leads will decrease. For infinitely large box, the lead tubes would be neutral, all charge variation would occur in the vicinity of the junction, and to ensure the neutrality of the system, all four capacitance matrix coefficients would be expected to be equal in magnitude. In other words, in the equivalent circuit diagram, only one capacitance matrix element describing the tube-tube interaction would remain. ${ }^{39}$

As more and more of the $(5,5)$ nanotube is inserted into the $(12,12)$ tube, the capacitance of the system increases and ultimately the system behaves similar to the case of two nested nanotube shells. This is shown in Fig. 9. The slope in this figure approaches a value of $0.012 \mathrm{aF}$, which is in reasonable agreement with the $0.015 \mathrm{aF}$ result obtained for the nanotube shells. Finally, we note that these results are all more or less independent from the dangling bonds present on the $(12,12)$ tube, since saturating these bonds with hydrogen changes the results by less than $1 \%$.

Having discussed the capacitance results, we address the issue of the bound states for this system. For a perfect, infinite periodic nanotube, the only eigenstates present in the system are the Bloch states, which are continuous in energy. However, a semi-infinite tube may also have states of discrete energy localized at the end of the tube. Such bound states have been predicted, and their energies calculated for a $(5,5)$ capped tube. ${ }^{44}$ Localized states in carbon nanotube tips have also been observed experimentally in scanning tunneling microscopy experiments. ${ }^{45}$

From the standpoint of capacitance, only the rearrangements of charge in the continuum states should matter, as these are the only ones in contact with the reservoirs. But to calculate this correctly, one must take care of the bound states properly. For our systems, it is computationally too difficult to find the localized eigenstates directly, as this would entail solving the scattering problem for the system with no incoming/outgoing states. ${ }^{10}$ However, the symmetry 


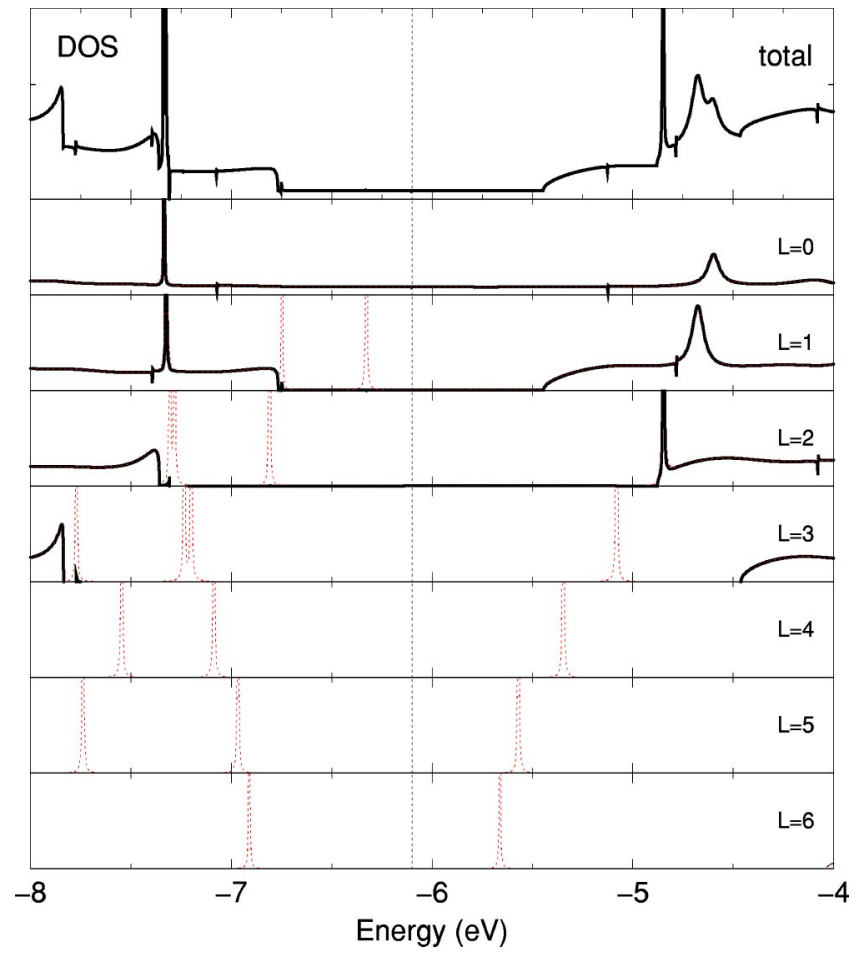

FIG. 10. (Color online) Total (top panel) and symmetry decomposed (lower panels) DOS (arbitrary units) for the $(12,12)$ open, semi-infinite tube. Note the presence of bound states, which appear for $\eta=5 \times 10^{-5}$ a.u. (dashed line). The Fermi energy is at $-6.10 \mathrm{eV}$ (dashed vertical line). Bound states are handled by keeping them either completely empty or fully occupied. For instance, to populate the bound state for the $L=6$ case, one needs to shift the contour integral limit to $E_{F}+0.82 \mathrm{eV}$. Alternatively, this state may be handled by choosing to terminate the contour integral limit for $L=6$ contribution below the energy of the unoccupied bound state, resulting in that bound state being empty. Note that in our selfconsistent calculation the energies of the bound states will shift depending on whether they are occupied or not.

decomposition of the DOS equation (24) allows the bound states to be readily identified. This is achieved by adding a complex parameter $i \eta$ to the denominator of Eq. (23) and comparing the density $d n_{L} / d E$ for different values of $\eta$. In Fig. 10, we show $d n_{L} / d E$ calculated with vanishing $\eta$ and with small but finite $\eta$. We see that the two densities coincide closely, except for the appearance of additional very sharp peaks. These new peaks appear only inside the band gap. We identify these peaks in density as being due to bound states. An electron in any one of these bound states is trapped there permanently, since there are no lead states of the same symmetry and energy through which it could leave the system. The bound states are primarily associated with the dangling bonds at the open end of the tube. We find that saturating these dangling bonds with hydrogen greatly reduces the number of bound states in the system.

Bound states may be dealt with by using Eq. (27) and choosing the limit of contour integration such that they remain separated from the point where the contour touches the real axis. In this way, the bound states are counted as being either completely occupied or completely empty. For in- stance, from Fig. 10, it is evident that there are no bound states for the $L=0$ term of the charge density, so that a self-consistent treatment of these terms is straightforward. Terms with $L$ from $L=1$ to $L=6$, however, have varying numbers of bound states in the gap, which may either be treated as being occupied by shifting the limit of the contour integral of Eq. (22) to include them or simply to keep them empty. Note also that the position of these bound states can shift slightly in the presence of a bias voltage. Unfortunately, the appropriate shifts in the contour limits, when these problematic states are present, is somewhat arbitrary, but appears to be unavoidable within the model. Generally, we have used the smallest possible shifts to eliminate the problem. Of course, in a real system the occupation of the bound states will largely depend on the way the system was prepared, or by the inclusion of additional interactions.

To summarize our treatment of the bound states, we note that our main purpose is to investigate the capacitance, which depends on charge rearrangements in the continuous states. To that end, we need to keep the bound states at a constant occupation (either full or empty) as a bias voltage is applied. The latter is accomplished by shifting the limits of the contour integral for the symmetry decomposed Green's functions. Without such a treatment, it was found to be difficult to have proper numerical convergence.

\section{Junction with conductance gap}

So far, we have examined the capacitance properties of well-separated nanotubes. Here, we examine the properties of a nanotube junction where two different metallic tubes are joined together, and are characterized by a conductance gap about the Fermi energy. Such a gap may arise if the bands at the Fermi energy of the two tubes making up the junction have a different symmetry. Such junctions are important as they form the basis of a number of nanodevices. ${ }^{40,41,42}$ The specific junction we consider is that of two semi-infinite $(12,0)$ and $(6,6)$ tubes, as shown in Fig. 11. The junction is symmetric under a rotation of $2 \pi / 6$ around the nanotube axis and, hence, obeys the $C_{6}$ rotation group symmetry. From Fig. 4 , it is clear that the $(12,0)$ Fermi energy bands have $L$ $=2$ symmetry, while the $(6,6)$ tubes have $L=0$ symmetry. This implies that for an electron arriving at the junction via a propagating state with energy close to the Fermi energy, there are no propagating states of the same symmetry for the electron to exit by. Because the junction does not break the sixfold symmetry, the electron cannot change its symmetry index by scattering at the junction. Hence, the electron cannot propagate into the other tube, and will therefore undergo total internal reflection at the junction. Note that although no scattering states are allowed across the junction, evanescent waves are not forbidden. These, however, decay exponentially away from the junction, and here appear to make a negligible contribution. Figure 12 illustrates the DOS and the conductance of the tube. ${ }^{43}$

It is interesting to consider the capacitance of such a junction as a possible element in a nanodevice. Note that if the perfect symmetry of this nanotube junction is broken, as is the case for asymmetric junctions, then the conductance gap 


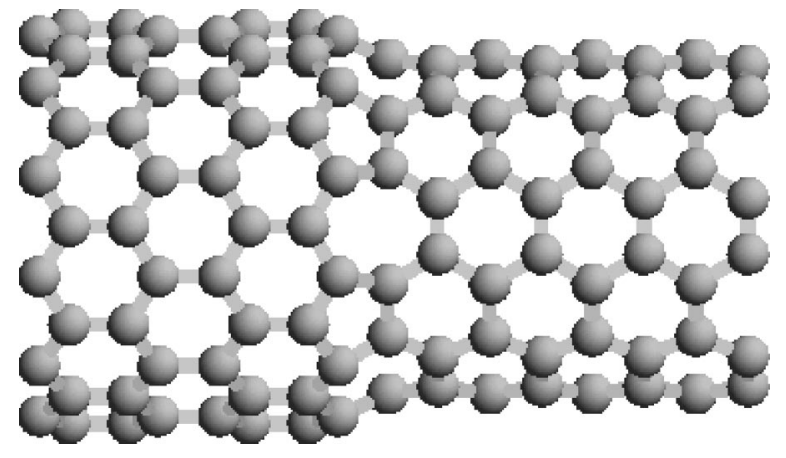

FIG. 11. Structure of $(12,0)(6,6)$ junction. Note the symmetry arrangement of (5-7) pairs about the circumference of the tubes, directly at the junction.

would disappear and current would flow directly through the device. In this case, it is no longer meaningful to define the device properties in terms of capacitance coefficients. Results as a function of bias were obtained for a junction consisting of five 48-atom unit cells for each side of the junction, i.e., 480 carbon atoms in total. A box of $40 \times 40$ $\times 44 \AA^{3}$ discretized by a grid of $256 \times 256 \times 256$ was used to describe the system. Some attention has also been paid to the length dependence of the junction capacitance by extending the length of $(6,6)$ tube out to eight unit cells for a total of 624 carbon atoms in total.

The Fermi energies of the tubes are -6.090 and $-6.103 \mathrm{eV}$ for the neutral $(6,6)$ and $(12,0)$ tubes, respectively. We could therefore, in principle, take $-6.097 \mathrm{eV}$ as the zero point for the electrochemical potential which is as close as possible to perfect neutrality. However, the $(12,0)$ nanotube is characterized by a tiny gap of $0.082 \mathrm{eV}$. Since we wish to focus on the metallic behavior of the tubes, we have opted to shift the electrochemical potential relative to the gate by applying a $+3 \mathrm{eV}$ to both potentials, which puts the equilibrium potential at $-3.078 \mathrm{eV}$. This shift also moves the band structure of the tubes, so that a neutral tube would be obtained by filling bands up to $-3.330 \mathrm{eV}$ for the $(6,6)$ and $-3.367 \mathrm{eV}$ for the $(12,0)$ tube. Because the zero of the electrochemical potential is now higher, the tubes acquire additional charges: 2.297 and 2.277 electrons for the $(6,6)$ and $(12,0)$ tubes, respectively. This represents a change of about $1 \%$ for each carbon atom in the junction.

We now examine how the charge in the junction rearranges itself under a bias voltage. For this discussion, a symmetry decomposition is essential, because in this case the two nanotubes are in direct contact and the capacitance cannot be simply obtained by counting the charge on each tube. First, we note that imposing a finite bias on the tubes does not break the symmetry of the junction, and hence the conductance gap persists. Nonzero conductance will only occur when the bias is large enough as to bring bands of the same symmetry $L$ into alignment. We, however, will limit our discussion to the regime where this does not occur. Consider what happens when a positive bias is applied to the left $(12,0)$ tube. The resulting charge distribution is shown in Fig. 13: charge is injected into the $L=2$ states near the Fermi energy. Such states exist in the $(12,0)$ tube and decay some

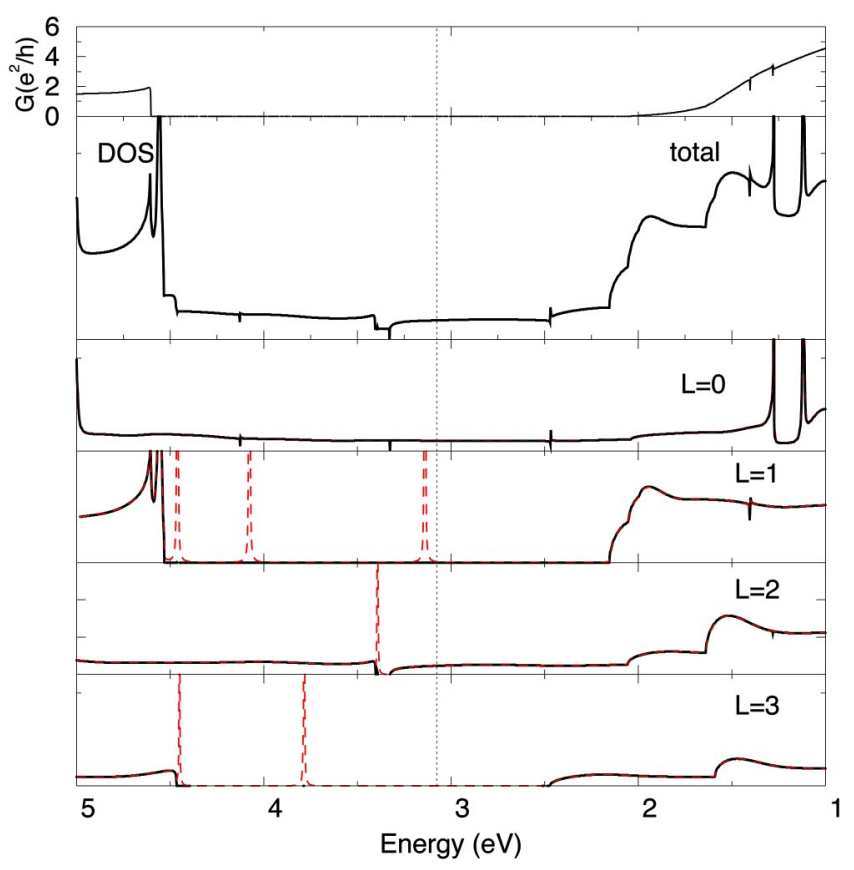

FIG. 12. (Color online) Conductance $G$ (top panel), total DOS (arbitrary units), and symmetry decomposed DOS (lower panels) for the $(12,0) /(6,6)$ junction. The electrochemical potential of the junction has been shifted by $+3.0 \mathrm{eV}$, so that the Fermi energy is located at $E_{F}=-3.078 \mathrm{eV}$ (dashed line in center). To correctly populate the bound states, the limits of the contour integrals were chosen to be at $E_{F}$ for $L=0,2,3$, and $E_{F}+0.272 \mathrm{eV}$ for $L=1$. The latter shifts compensate for slight shifts in the position of the bound states under a bias voltage.

distance into the $(6,6)$ tube. Upon injection, the induced charge with opposite sign appears in the $L=0$ states, which exist in the $(6,6)$ tube and decay some distance into the $(12,0)$ tube. Since each of these decaying components penetrates some distance into the other tubes, the charge component will overlap across the junction. Charges are induced in a similar fashion in the $L=2$ states of the $(12,0)$ tube if the bias is applied to the $(6,6)$ nanotube. The states with $L$ $=1,3$ symmetry do not have any conducting bands near the Fermi energy, and so these will behave like semiconductors, and have a limited polarization response. So, while this charge may undergo some rearrangement, the total charge associated with these states is more than two orders of magnitude smaller than the charge accumulated in the $L=0,2$ states, as shown in Fig. 14.

From the discussion, it is clear that the capacitance matrix coefficients may be calculated from the charge accumulation in the $L=0,2$ states: charge in the $L=0$ state could only have arrived through the $(6,6)$ tube, while charge in the $L=2$ states could only have arrived through the $(12,0)$ tube. Because filled bands cannot carry current, ${ }^{46}$ the $L=1,3$ states will not accumulate any charge and hence can be ignored. We label the voltage applied to the $(12,0)$ tube as $d V_{(12,0)}$, and that on the $(6,6)$ tube as $d V_{(6,6)}$. Similarly, $d Q_{0,2}$ corresponds to the changes in the $L=0,2$ charge, respectively. The charge accumulated in the junction for these two applied 


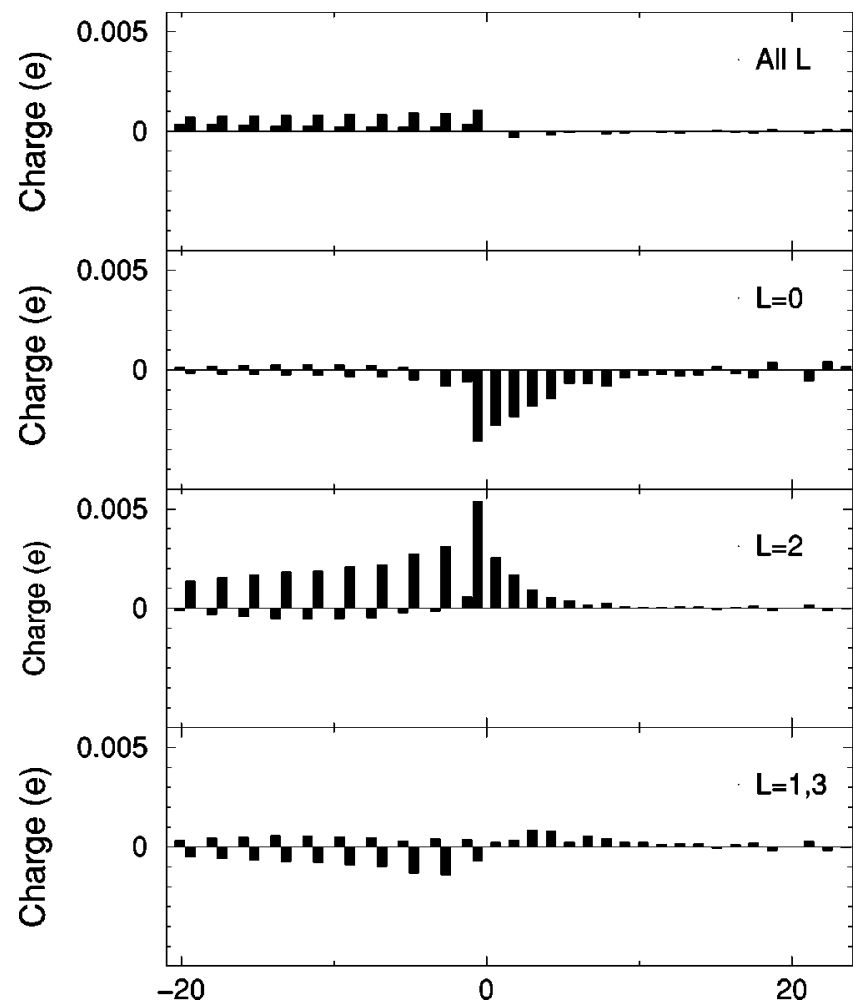

FIG. 13. Total and symmetry decomposed charge accumulation under a $+0.272 \mathrm{eV}$ bias applied to the left $(12,0)$ tube of the $(12,0)$ / $(6,6)$ nanotube junction.

voltages is plotted in Fig. 15, and note the linear dependence on the bias voltage. The capacitance coefficients, also plotted here, are then given by

$$
\begin{gathered}
C_{(6,6),(6,6)}=\frac{d Q_{0}\left(d V_{(6,6)}\right)}{d V_{(6,6)}}, \\
C_{(12,0),(6,6)}=\frac{d Q_{2}\left(d V_{(6,6)}\right)}{d V_{(12,0)}}, \\
C_{(6,6),(12,0)}=\frac{d Q_{0}\left(d V_{(12,0)}\right)}{d V_{(6,6)}}, \\
C_{(12,0),(12,0)}=\frac{d Q_{2}\left(d V_{(12,0)}\right)}{d V_{(12,0)}} .
\end{gathered}
$$

Hence, $C_{(6,6)(12,0)}$ gives the charge arriving into the system from the $(6,6)$ reservoir in response to an electrochemical change in the $(12,0)$ reservoir. The volume of integration in this case is the whole junction, i.e., all the $\mathrm{C}$ atoms within the simulation box. The small-bias capacitance coefficients calculated are

$$
\begin{gathered}
C_{(6,6)(6,6)}=0.205 \mathrm{aF}, \\
C_{(12,0)(6,6)}=-0.125 \mathrm{aF}, \\
C_{(6,6)(12,0)}=-0.127 \mathrm{aF}, \\
C_{(12,0)(12,0)}=0.199 \mathrm{aF} .
\end{gathered}
$$

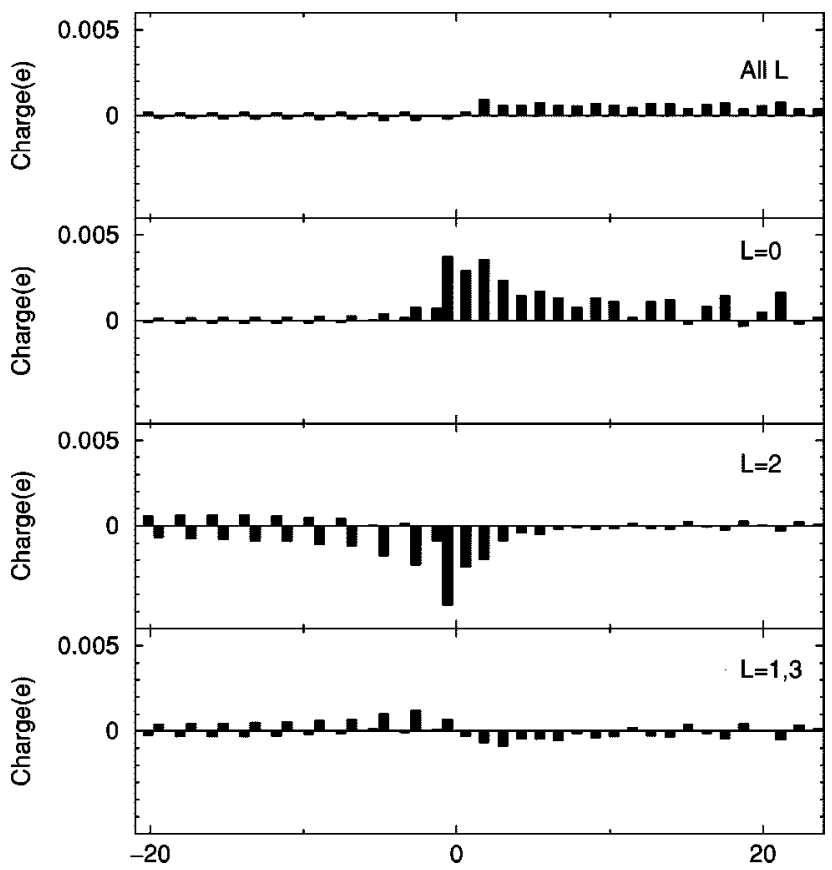

FIG. 14. Total and symmetry decomposed charge accumulation under a $+0.272 \mathrm{eV}$ bias applied to the right $(6,6)$ tube of the $(12,0) /$ $(6,6)$ nanotube junction.

These show behavior that is qualitatively similar to the coefficients as for the other nanotube systems. Thus, the selfcharging components are larger and depend linearly on the tube length because of the interaction with the surrounding box. We have checked this explicitly by calculating the capacitance coefficients as the number of $(6,6)$ unit cells is increased. As expected, all the capacitance coefficients remain constant except for the $C_{(6,6)(6,6)}$ coefficient which increases linearly. We argue as before that all terms of the capacitance matrix will tend to the same absolute value as the size of the container box is increased, and this will be accomplished mostly through a reduction in the self-charging terms. Therefore, we expect that $C_{(12,0),(6,6)}$ and $C_{(6,6),(12,0)}$ terms we have obtained for a finite box should be reasonably close to the corresponding coefficients for the junction in vacuum. $^{39}$

Perhaps the most important feature for this system is that the capacitance value obtained for this nanotube junction is very high, as compared to that of the nested nanotube shells. Indeed, units with such high capacitance values may turn out to be useful for memory devices. We believe that this feature is due to the overlapping DOS for the induced charges, which boosts the capacitance even though the actual contact area is relatively small. Finally, we note that the investigated $(12,0) /(6,6)$ junction is perhaps the simplest system with a conductance gap, and that other similar junctions exist. An $(n, m)$ tube has $J$-fold rotational symmetry if both $n$ and $m$ are divisible by $J$. The necessary condition to be able to form a symmetric junction is that the helicity indices of the second tube all have a common divisor with $J$. For such a system, a conductance gap may be the result depending on the symmetry of states in both tubes at the Fermi energy. A particularly interesting configuration would involve a system of two 

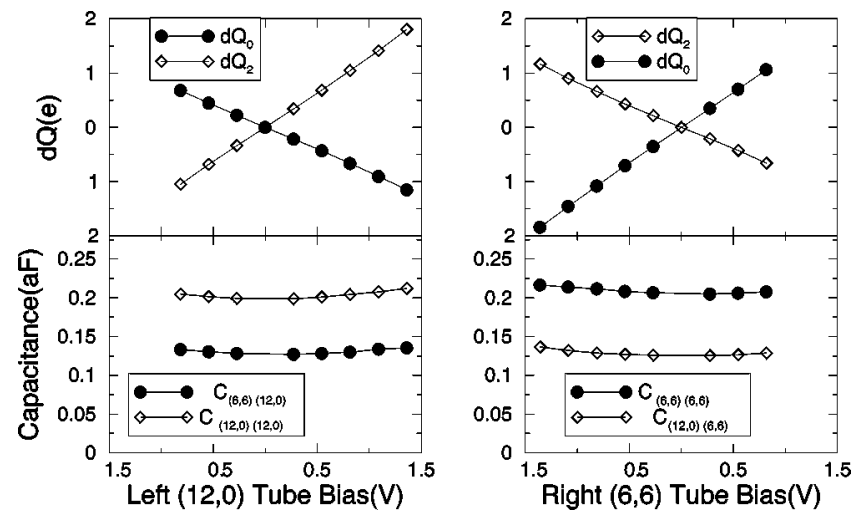

FIG. 15. Charge accumulation (top panels) and capacitance coefficients (lower panels) for the biased $(12,0) /(6,6)$ junction. For the left (right) panels, the bias is applied to the $(12,0)$ tube $[(6,6)$ tube]. Note that charge accumulation is quite linear and the capacitance coefficients more or less are constant over the applied bias-voltage regime.

tubes where a conductance gap is present, but where one tube is sufficiently larger than the other, so that direct insertion of one tube into the other is possible. Such a system would most likely see an extra enhancement in the capacitance because it combines both the boost from the insertion with the effect of the overlapping DOS from the junction.

\section{Nanotubes as scanning capacitance probes}

Carbon nanotubes may well have a considerable future as capacitance probes, and, indeed, the first experiments of this type are beginning to emerge. ${ }^{15}$ Hence, as a prototypical example, we have investigated the capacitance properties of a capped $(5,5)$ nanotube over a flat (100) Al surface, as shown in Fig. 16. The simulation box size considered was 60 $\times 60 \AA^{2}$ and enclosed $3 \times 3,5 \times 5$, or $9 \times 9$ Al electrodes. The $9 \times 9$ system, which was the largest practical system we could investigate, consisted of $162 \mathrm{Al}$ and 150 carbon atoms. Typical capacitance calculations for this system took about $24 \mathrm{~h}$ on eight processors of a small Pentium IV cluster.

In our investigations, we focused primarily on the charging coefficients as a function of the distance from the Al surface, with the bias applied to the nanotube only. The closest approach between the nanotube probe and the surface considered was about $7.3 \AA$, so that again there was no flow of current between the two components. At this distance, the charge accumulation is linear to within $1 \%$ for up to $\pm 0.3 \mathrm{~V}$, allowing for the extraction of well-defined capacitance coefficients. At this distance, we measured

$$
\begin{gathered}
C_{(9 \times 9)(5,5)}=-0.0186 \mathrm{aF}, \\
C_{(5,5)(5,5)}=0.0777 \mathrm{aF} .
\end{gathered}
$$

As may be expected, the self-charging coefficient is very much larger than the cross-charging term. This is due to the strong interaction of the nanotube with the surrounding box, and the relatively large distance between the nanotube and the Al surface. The actual charge distribution is shown in Fig. 16. Several expected features are evident. On the nano-
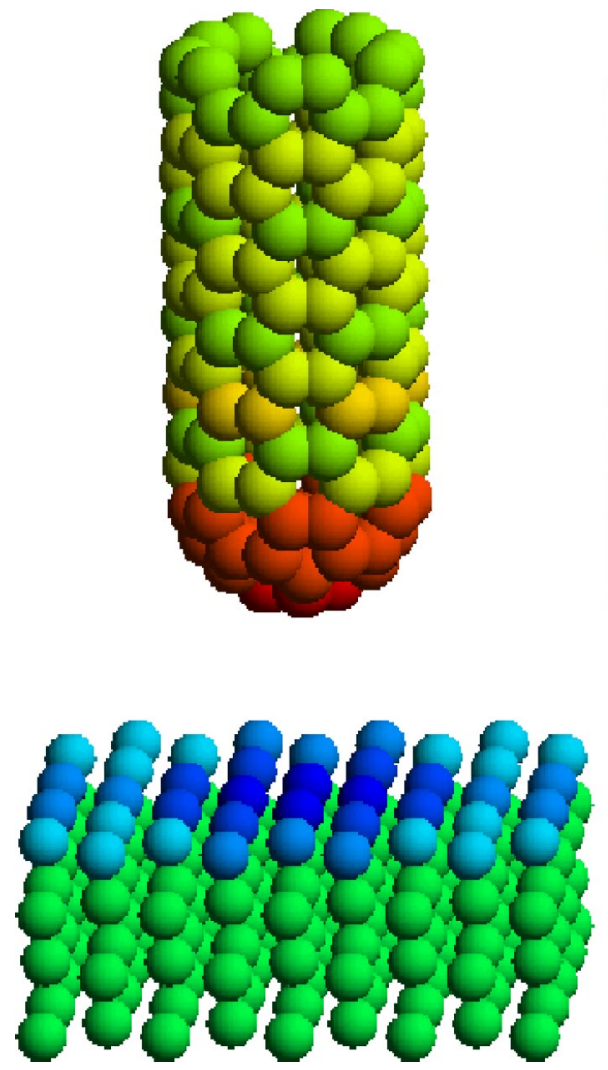

FIG. 16. (Color online) Accumulated charge on the $9 \times 9 \mathrm{Al}$ electrode and $(5,5)$ capped nanotube when a positive bias is applied to the tube. Note that the charge accumulated on the electrode in response to the positive charging of the nanotube is concentrated in the middle of top electrode layer.

tube, most of the charge is located right at the tip, while the charge on the Al surface is well localized directly underneath the charged nanotube in the middle of the electrode.

Figure 17 summarizes the capacitance measurements for the nanotube over different electrodes as a function of increasing distance. As expected, the cross-charging capacitance increases as the size of the Al surface becomes larger and decreases as the distance between the nanotube and the surface is increased. To estimate the capacitance for the case of a nanotube over an infinite-sized Al surface, we have carried out a finite-size scaling analysis. As shown in the inset, there is almost perfect scaling with the value of the capacitance coefficients versus $1 / L^{2}$. The estimated capacitance coefficient at the distance of closet approach is $0.0201 \mathrm{aF}$, which is only up slightly from the $9 \times 9$ result. As a function of distance, the data are well described by the fit

$$
C_{(\infty \times \infty)(5,5)}(d)=(0.197 / d-0.0069) \mathrm{aF} .
$$

These capacitance coefficients are actually quite low, and for scanning capacitance applications one may well want to increase the charging response. One obvious way to achieve this is to functionalize the ends of the nanotube, say, with small metal clusters. To test this idea, we have placed an $\mathrm{Al}$ atom on the nanotube axis both $3.28 \AA$ inside and $1.65 \AA$ outside the nanotube cap. When the $\mathrm{Al}$ atom is placed inside 


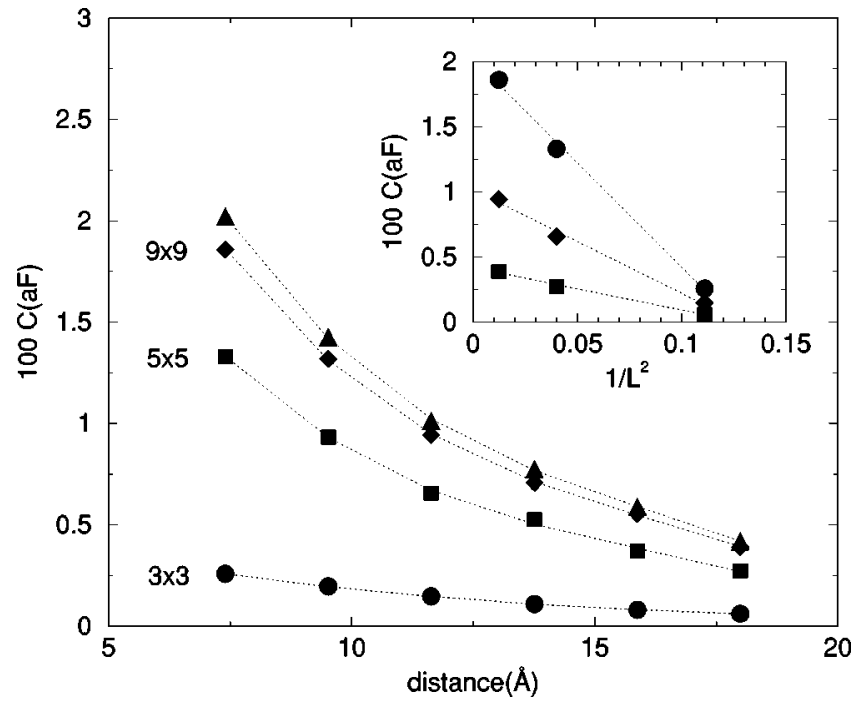

FIG. 17. Cross capacitance as a function of separation distance for $3 \times 3,5 \times 5$, and $9 \times 9 \mathrm{Al}$ electrodes. The extrapolated infinite width electrode result is included for comparison (triangles). The inset shows examples of fitting used to obtain the infinite width limit, with sample curves for three different distances shown.

the tube, the calculated capacitance is $-0.0192 \mathrm{aF}$, which is essentially the same as for the system without the $\mathrm{Al}$ atom. This suggests that small metal clusters placed inside the nanotube are unlikely to enhance the response of the system, because of the screening effects. Placing the $\mathrm{Al}$ atom outside the nanotube gives a capacitance coefficient of $-0.022 \mathrm{aF}$, which represents a $14 \%$ increase in the response. Hence, placing small metal clusters on the nanotube tip is likely to have significantly enhanced the charging effects. As a further test, we have also investigated the effects of placing an oxygen atom (electron acceptor) outside the nanotube cluster. However, in this situation, the effect was similar to adding an $\mathrm{Al}$ atom to the nanotube. The measured capacitance coefficient was $-0.0199 \mathrm{aF}$, so that response is somewhat lower than adding an $\mathrm{Al}$ atom. This difference presumably reflects the chemistry and DOS near the Fermi energy for the oxygen-doped system. Finally, for completeness, we show in Fig. 18 the induced charges for the different probe systems examined, with the positions of the dopant atoms marked. Note that for all the systems, the charges induced in the Al surface are primarily localized within the top layer only; as with previously discussed systems, there is evidence for considerable self-charging effects all the way along the entire length of the nanotube.

\section{SUMMARY}

In summary, we have computed the finite-bias capacitance coefficients for prototypical carbon nanotube systems, using a recently developed DFT-NEGF approach. Specific systems considered included nested two- and three-shell carbon nanotubes, the insertion of one nanotube into another, a nanotube junction with a conductance gap, and the behavior of a nanotube as a capacitance probe. Generally speaking, these systems consisted of several hundreds of atoms each. Hence, in



FIG. 18. (Color online) Charging profiles as various impurities are added to the system. From top to bottom, we illustrate the case of no impurities present, an $\mathrm{Al}$ atom inside the tube, an $\mathrm{Al}$ atom outside and in front of the tube, and an $\mathrm{O}$ atom in front of the tube.

order to cut down on the computational costs and to better analyze the induced charges, a symmetry decomposition of the charge density matrix in terms of the irreducible elements of the rotational group of the nanotube about its axis was found to be particularly useful. For each of the systems considered, we provide estimates of the capacitance coefficients and an analysis of the induced charges. While the capacitance for most systems is small (attofarad range), its value may be boosted by nesting and/or inserting nanotubes into each other. The capacitance of the $(12,0) /(6,6)$ was also considerably larger than that of the other systems, which is an effect that most likely derives from the overlapping DOS of each junction element. Quantum effects are clearly evident for these nanotube systems, and derive mostly from the finite DOS available. Hence, the notion of the electrochemical capacitance is essential for understanding this important nanoscale system.

\section{ACKNOWLEDGMENTS}

We thank Jeremy Taylor and Brian Larade for help in the early stages of this work. We gratefully acknowledge financial support from NSF and U.S. DOE (C.R.), NSERC of Canada (H.G.), and a RGC grant (Grant No. HKU 7091/01P) from the SAR Government of Hong Kong (J.W.). We would like to thank the North Carolina Supercomputing Center (NCSC) for extensive computer time.

\section{APPENDIX}

Here we outline calculations of the quantum corrections for the capacitance using the linear-response theory of Büttiker. ${ }^{3}$ It is assumed that the system consists of a number of quantum conductors, each connected to its own reservoir with its own electrochemical potential, so that the initial electrostatic potential $U\left(\left[\mu_{\alpha}\right], \mathbf{r}\right)$ is a complicated functional both of the position vector and the electrochemical potentials. A small variation $d \mu_{\alpha}$ in one of the electrochemical 
potentials brings the system to a new equilibrium state, such that $\quad e U\left(\left[\mu_{\alpha}+d \mu_{\alpha}\right], \mathbf{r}\right)-e U\left(\left[\mu_{\alpha}\right], \mathbf{r}\right)=e d U\left(\mu_{\alpha}, \mathbf{r}\right)$ $=\Sigma_{\alpha} u_{\alpha} d \mu_{\alpha}$, where $u_{\alpha}$ is the characteristic potential,

$$
u_{\alpha}=\left.e \frac{d U\left(\left[\mu_{\alpha}\right], \mathbf{r}\right)}{d \mu_{\alpha}}\right|_{d \mu_{\alpha}=0} .
$$

Charge rearranges itself in two different ways when the electrochemical potential of a reservoir changes. First, there is the injected charge which is written in terms of filling up of the DOS for the incoming charge carriers:

$$
d \rho_{i n j, \alpha}(\mathbf{r})=\frac{d n(\mathbf{r}, \alpha)}{d E} d \mu_{\alpha} .
$$

The injectivity is $d n(\mathbf{r}, \alpha) / d E$, and gives the partial density of states associated with carriers arriving to $\mathbf{r}$ from reservoir $\alpha$ after a variation $d \mu_{\alpha}$. The sum over all the injectivities from the different reservoirs gives the total local density of states. Second, in response to the injected charge, there is the induced charge density generated by the change in the electrostatic potential inside the device. Within the ThomasFermi approximation the induced density is then related to the potential via

$$
d \rho_{\text {ind, } \alpha}(\mathbf{r})=-\sum_{\alpha} \frac{d n(\alpha, \mathbf{r})}{d E} e d U(\mathbf{r})=-\frac{d n(\mathbf{r})}{d E} u_{\alpha} d \mu_{\alpha},
$$

with $d n(\alpha, \mathbf{r}) / d E$ representing the emissivity, i.e., the partial density of states associated with carriers ejected into the reservoir $\alpha$ from point $\mathbf{r}$ in response to a change in the electrochemical potential. Generally, in the absence of magnetic fields, the injectivity and emissivity are the same. The characteristic potential is then obtained self-consistently by solving the modified Poisson's equation

$$
-\nabla^{2} u_{\alpha}(\mathbf{r})=4 \pi e^{2}\left(\frac{d n(\mathbf{r})}{d E} u_{\alpha}-\frac{d n(\mathbf{r}, \alpha)}{d E}\right) .
$$

Once $u_{\alpha}$ is known, the sum of the injected and induced charges integrated over the volume of the conductor may be found. This gives the charge accumulation $d Q_{\alpha}$ on conductor $\alpha$ due to changes in the electrochemical potential in reservoir $\beta$. The capacitance matrix coefficients are then given by

$$
C_{\alpha \beta}=\frac{e d Q_{\alpha}}{d \mu_{\beta}}=e^{2} \int d \mathbf{r}\left[\delta_{\alpha \beta} \frac{d n(\mathbf{r}, \alpha)}{d E}-\frac{d n(\alpha, \mathbf{r})}{d E} u_{\beta}(\mathbf{r})\right] .
$$

We now have all the ingredients for calculating the capacitance coefficients for simple geometrical situations. The simplest case of a parallel-plate capacitor is described at length in Ref. 3. In what follows, we will extend our analysis to concentric cylindrical capacitors, without any edge effects.

Consider two concentric cylinders of radius $R_{1}$ and $R_{2}$. Each cylinder is assumed to be thin and unable to screen its interior; the value of the potential insider the cylinder walls is assumed to be constant. Equal and opposite charges will reside on each of the cylinders, so that the total charge is zero. Hence, $u_{1}(\rho)$, using cylindrical coordinates $\rho$, satisfies the cylindrical Poisson equation

$$
\frac{1}{\rho} \frac{\partial}{\partial \rho}\left[\rho \frac{\partial u(\rho)}{\partial \rho}\right]=0
$$

This is easily solved to obtain $u(\rho)=a_{2}+a_{3} \ln (\rho)$ for $R_{1}$ $<\rho<R_{2}$ and $u(\rho)=a_{1}$ for $\rho<R_{1}$ and $u(\rho)=a_{4}$ for $\rho$ $>R_{2}$. One can solve for the coefficients $a_{i}$ using the fact that $u_{1}(\rho)$ is continuous at the walls, and its derivative discontinuous due to the presence of the sheet charge density in the cylinder. The accumulated sheet charge density is proportional to $\left(1-u_{1}\right) d \sigma_{1} / d E$ on cylinder 1 and $u_{1} d \sigma_{2} / d E$ on cylinder 2 , where $d \sigma_{1,2} / d E$ are the respective densities of states per unit area. The capacitance coefficients per unit length are then obtained by inspection:

$$
\frac{2 \pi l}{C}=\ln \left(R_{2} / R_{1}\right)+\lambda_{1} / R_{1}+\lambda_{2} / R_{2},
$$

with $\lambda_{1,2}^{-1}=4 \pi e^{2} d \sigma_{1,2} / d E$, which is the formula quoted in the main text.
${ }^{1}$ See, for example, Classical Electrodynamics, 2nd ed., edited by J. D. Jackson (Wiley Press, New York, 1975).

${ }^{2}$ T.P. Smith, B.B. Goldberg, P.J. Stiles, and M. Heiblum, Phys. Rev. B 32, 2696 (1985); T.P. Smith, W.J. Wang, and P.J. Stiles, ibid. 34, 2995 (1986).

${ }^{3}$ M. Büttiker, H. Thomas, and A. Petre, Phys. Lett. A 100A, 364 (1993); M. Büttiker, J. Phys.: Condens. Matter 5, 9361 (1993).

${ }^{4}$ T. Christen and M. Büttiker, Phys. Rev. Lett. 77, 143 (1996).

${ }^{5}$ J. Wang, H. Guo, J.L. Mozos, C.C. Wan, G. Taraschi, and Q. Zheng, Phys. Rev. Lett. 80, 4277 (1998).

${ }^{6}$ J.G. Hou, B. Wang, J. Yang, X.R. Wang, H.Q. Wang, Q. Zhu, and X. Xiao, Phys. Rev. Lett. 86, 5321 (2001).

${ }^{7}$ S. Datta, Electronic Transport in Mesoscopic Systems (Cambridge University Press, Cambridge, 1995).
${ }^{8}$ D.K. Ferry, and S.M. Goodnick, Transport in Nanostructures (Cambridge University Press, Cambridge, 1997).

${ }^{9}$ J. Taylor, H. Guo, and J. Wang, Phys. Rev. B 63, 121104(R) (2001).

${ }^{10}$ J. Taylor, H. Guo, and J. Wang, Phys. Rev. B 63, 245407 (2001).

${ }^{11}$ See, for example, M.S. Dresselhaus, G. Dresselhaus, and P. C. Eklund, Science of Fullerenes and Carbon Nanotubes (Academic Press, New York, 1996); J. Bernholc, C. Roland, and B. Yakobson, Crit. Rev. Solid State Mater. Sci. 2, 706 (1997); J. Bernholc, D. Brenner, M. Buongiorno Nardelli, V. Meunier, and C. Roland, Annu. Rev. Mater. Res. 32, 347 (2002).

${ }^{12}$ See, for example, S. Frank, P. Poncharal, Z.L. Wang, and W.A. deHeer, Science 280, 1744 (1998); A. Bezryadin, A.R.M. Verschueren, S.J. Tans, and C. Dekker, Phys. Rev. Lett. 80, 4036 
(1998); S.J. Tans, M.H. Devoret, H.J. Dai, A. Thess, and R.E. Smalley, Nature (London) 386, 474 (1997); M. Bockrath, D.H. Cobden, J. Lu, A.G. Rinzler, and R.E. Smalley, ibid. 397, 598 (1998); R. Martel, T. Schmidt, H.R. Shea, T. Hertel, and Ph. Avouris, Appl. Phys. Lett. 73, 2447 (1998).

${ }^{13}$ See, for example, L. Chico, L.X. Benedict, S.G. Louie, and M.L. Cohen, Phys. Rev. B 54, 2600 (1996), and many other papers.

${ }^{14}$ Y.P. Zhao, B.-Q. Wei, P.M. Ajayan, G. Ramanath, T.-M. Lu, G.-C. Wang, A. Rubio, and S. Roche, Phys. Rev. B 64, 201402 (2001).

${ }^{15}$ S.V. Kalinin, D.A. Bonnell, M. Freitag, and A.T. Johnson, Appl. Phys. Lett. 81, 754 (2002); 81, 5219 (2002).

${ }^{16}$ C. Roland, M. Buongiorno Nardelli, J. Wang, and H. Guo, Phys. Rev. Lett. 84, 2921 (2000).

${ }^{17}$ P. Pomorski, C. Roland, H. Guo, and J. Wang, Phys. Rev. B 67, 161404(R) (2003).

${ }^{18}$ A.P. Jauho, N.S. Wingreen, and Y. Meir, Phys. Rev. B 50, 5528 (1994).

${ }^{19}$ B.G. Wang, J. Wang, and H. Guo, Phys. Rev. Lett. 82, 398 (1999).

${ }^{20}$ C. Roland, B. Larade, J. Taylor, and H. Guo, Phys. Rev. B 65, 041401(R) (2002).

${ }^{21}$ B. Larade, J. Taylor, Q.R. Zheng, H. Mehrez, P. Pomorski, and H. Guo, Phys. Rev. B 64, 195402 (2002).

${ }^{22}$ M. Brandbyge, J.L. Mozos, P. Ordejon, J. Taylor, and K. Stokbro, Phys. Rev. B 65, 165401 (2002).

${ }^{23}$ B. Larade, J. Taylor, H. Mehrez, and H. Guo, Phys. Rev. B 64, 075420 (2001).

${ }^{24}$ C.C. Kaun, B. Larade, H. Mehrez, J. Taylor, and H. Guo, Phys. Rev. B 65, 205416 (2002).

${ }^{25}$ C. Roland, V. Meunier, B. Larade, and H. Guo, Phys. Rev. B 66, 035332 (2002).

${ }^{26}$ J. Taylor, M. Brandbyge, and K. Stokbro, Phys. Rev. Lett. 89, 138301 (2002).

${ }^{27}$ C.C. Kaun, B. Larade, and H. Guo, Phys. Rev. B 67, 121411 (2003).

${ }^{28}$ A. Brandt, Math. Comput. 31, 333 (1977).

${ }^{29}$ O.F. Sankey and D.J. Niklewski, Phys. Rev. B 40, 3979 (1989).

${ }^{30}$ The NEGF-DFT code also supports $d$ orbitals. However, for the atoms discussed in this paper, they are not needed.

${ }^{31}$ D.R. Hamann, M. Schlütter, and C. Chiang, Phys. Rev. Lett. 43, 1494 (1982).
${ }^{32}$ S. Sanvito, C.J. Lambert, J.H. Jefferson, and A.M. Bratkovsky, Phys. Rev. B 59, 11936 (1999).

${ }^{33}$ R. Tamura, Phys. Rev. B 64, 201404 (2001).

${ }^{34}$ D. M. Bishop, Group Theory and Chemistry (Clarendon Press, Oxford, 1973).

${ }^{35}$ A short summary of the results for nested two-shell tubes, a $(5,5)$ tube inserted into a $(12,12)$ tube, and the nanotube junction have previously been published in Ref. 17.

${ }^{36}$ J. Cumings and A. Zettl, Science 289, 602 (2000).

${ }^{37}$ G. Chen, S. Bandow, E.R. Margine, C. Nisoli, A.N. Kolmogorov, V.H. Crespi, R. Gupta, G.U. Sumanseskere, S. Iijima, and P.C. Eklund, Phys. Rev. Lett. 90, 257403 (2003).

${ }^{38} \mathrm{~A}$ complete and correct modeling of the polarization response of the semiconducting nanotube is a difficult problem that may well require the inclusion of $d$ orbitals, and higher. Here, our aim is simply to illustrate the dielectric effect of an intermediate tube, which is included at the level of $s$ and $p$ orbitals. A more correct modeling and treatment of polarizability will be left to future investigations.

${ }^{39} \mathrm{We}$ also observe in both the inserted nanotube system and the nanotube junction long-ranged Friedel-type oscillations which do not completely decay away within our central simulation box. Such oscillations are only seen in $d Q$; the equilibrium charge $Q$ does not display such oscillations. This behavior does not affect the value of capacitance coefficients in any significant way. Specifically, the capacitance coefficients do not fluctuate with these oscillations as the size of the box is varied.

${ }^{40}$ L. Chico, V.H. Crespi, L.X. Benedict, S.G. Louie, and M.L. Cohen, Phys. Rev. Lett. 76, 971 (1996).

${ }^{41}$ Z. Yao, H.W.C. Postma, P. Yang, and C.M. Lieber, Nature (London) 402, 273 (1999).

${ }^{42}$ D. Orlikowski, M. Buongiorno Nardelli, J. Bernholc, and C. Roland, Phys. Rev. Lett. 83, 4132 (1999).

${ }^{43}$ D. Orlikowski, M. Buongiorno Nardelli, J. Bernholc, and C. Roland, Phys. Rev. B 61, 14194 (2000).

${ }^{44}$ Ryo Tamura and Masaru Tsukada, Phys. Rev. B 52, 6015 (1995).

${ }^{45}$ D.L. Carroll, P. Redlich, P.M. Ajayan, J.C. Charlier, X. Blase, A. de Vita, and R. Car, Phys. Rev. Lett. 78, 2811 (1997).

${ }^{46}$ N.W. Ashcroft and N.D. Mermin, Solid State Physics (Saunders, Philadelphia, 1976). 